\title{
THE COEFFICIENT PROBLEM IN THE THEORY OF LINEAR PARTIAL DIFFERENTIAL EQUATIONS $\left({ }^{1}\right)$
}

\author{
BY \\ STEFAN BERGMAN
}

1. The basic idea of the application of integral operators to the Weierstrass-Hadamard direction. In order to generate and investigate solutions of differential equations, operators $\mathbf{p}$ (defined as the integral operators of the first kind) have been introduced in $[2 ; 6]\left({ }^{2}\right)$. $p$ transforms analytic functions of one and two variables into solutions of linear elliptic differential equations of two and three variables, respectively. It has been shown in the abovementioned papers that $\mathbf{p}$ (as well as some other operators connected with $\mathbf{p}$ ) preserves many properties of the functions to which the operator is applied. This situation permits us to use theorems in the theory of functions to obtain theorems not merely on harmonic functions in two variables, but on solutions of other linear differential equations as well $\left({ }^{3}\right)$.

In the present paper the above-mentioned method is used to prove connections between the properties in the large of solutions $\psi$ of certain linear differential equations, see (1.1) and (1.3), on one side and the structure of certain subsequences of the coefficients of the series development of $\psi$ at the origin on the other.

Let us formulate these procedures in a somewhat more concrete manner, at first for equations in two variables. Let $\psi$ be a (real) solution of the differential equation

$$
\begin{gathered}
L(\psi) \equiv \psi_{z z^{*}}+F\left(z, z^{*}\right) \psi \equiv \frac{1}{4}\left(\psi_{x_{1} x_{1}}+\psi_{x_{2} x_{2}}\right)+\tilde{F}\left(x_{1}, x_{2}\right) \psi=0, \\
z=x_{1}+i x_{2}, \quad z^{*}=x_{1}-i x_{2},
\end{gathered}
$$

where $F\left(z, z^{*}\right)=\tilde{F}\left(x_{1}, x_{2}\right)$ is a (real) entire function $\left({ }^{4}\right)$.

In analogy to the case of harmonic functions we associate with $\psi$ an analytic function $g$ of one complex variable $z$ by the relation

$$
\psi(z, 0)=(g(z)+\bar{g}(0)) / 2 .
$$

Received by the editors August 17, 1951.

(1) Paper done under contract with the Office of Naval Research N5ori 76/16 NR 043-046.

(2) Numbers in brackets refer to the bibliography at the end of the paper.

$\left(^{3}\right)$ The operation $\operatorname{Re}[p()]$ represents a generalization to the case of more general linear differential equations of the operator $\operatorname{Re}[$ ] (taking of real part). It permits us to generalize methods in the theory of harmonic functions which are based on the use of the operator Re. A subscript attached to an operator indicates dimension of the function produced.

${ }^{(4)}$ I.e., real for real values of the arguments $x_{1}, x_{2}$, and entire when continued to complex values of $x_{1}, x_{2}$. 
(Thus $g / 2+$ const. coincides with $\psi$ in the so-called characteristic plane, $z^{*}=0$.) $g(z)$ is defined as the $\mathrm{C}$-associate of $\psi\left(z, z^{*}\right)$ with reference to the origin. $\operatorname{Re}[\mathbf{p}()]$, where $\mathbf{p}=\mathbf{p}_{2}$ is the operator of the first kind mentioned above, transforms $g(z)$ back into the solution $\psi\left(z, z^{*}\right)$.

The expression $\operatorname{Re}\left[\mathrm{p}_{2}(g)\right]$, representing the solution $\psi$ in terms of its C-associate, $g$, involves two integrations, see (2.7), (2.5). The first integration, see (2.7), transforms $g$ into a function of one complex variable. Due to this fact various classical results on the relations between analytic functions (in particular, algebraic functions) on one side, and their integrals on the other, can be interpreted successfully as theorems on solutions $\psi$ of (1.1). Using these and other properties of $\mathbf{p}_{2}$ the classical results in the theory of functions yield among others the following types of theorems:

Let $R_{0}$ be the Riemann surface of an algebraic function, and let $\mathfrak{A}\left(\mathbb{R}_{0}\right)$ be the class of solutions of (1.1) such that the $\mathbf{C}$-associates $2 \psi(z, 0), \psi \in \mathfrak{A}\left(R_{0}\right)$, are single-valued on $\mathbb{R}_{0}$. Then every $\psi \in \mathfrak{A}\left(\mathbb{R}_{0}\right)$ can be represented in the form of an integral, whose integrand is a product of two expressions. The first factor depends only on $F$ (i.e., only upon the equation), the second depends only upon $R_{0}$ (i.e., independent of $F$ ) and (for all $\psi \in \mathfrak{A}\left(\mathcal{R}_{0}\right)$ ) can be represented in a closed form involving algebro-logarithmic expressions, certain $\theta$-functions, their derivatives, and finitely many transcendental functions $\left({ }^{5}\right)$. Using the classical theorems (Weierstrass, Hadamard, Eisenstein, Pólya, Szegö, ...) it is possible to show that the subsequences $\left\{a_{m 0}\right\}, m=0,1,2$, $\cdots$, of the developments $\psi=\sum_{m, n} a_{m n} z^{m} z^{* n}, \psi \in \mathfrak{A}\left(\mathcal{R}_{0}\right)$ lie in a certain manifold (which is independent of $F$ ) of the coefficient space $(\S \S 2,3)$.

The study of differential equations in three variables represents a further step in the development of this approach $\left({ }^{6}\right)$. In continuation of $[6 ; 8]$, in the present paper we consider differential equations

$$
\begin{gathered}
\left(\partial^{2} \psi / \partial x_{1}^{2}\right)+\left(\partial^{2} \psi / \partial x_{2}^{2}\right)+\left(\partial^{2} \psi / \partial x_{3}^{2}\right)+F \psi=0, \\
\psi=\psi(X), \quad X=\left(x_{1}, x_{2}, x_{3}\right),
\end{gathered}
$$

where $F$ is an entire function of $r^{2}=\sum_{\nu=1}^{3} x_{\nu}^{2}$. These investigations consist of two steps: the study (1) of the mappings of harmonic functions $\psi(X)$, onto algebras, (2) of the transition from the harmonic functions to the solutions of (1.3) (with $F \neq 0$ ).

(2) proceeds in essentially the same manner as in the two-dimensional case, and involves mainly technical difficulties. Thus problem (1) is the major point in this study.

In $\$ 4$ we discuss the mappings of harmonic functions onto functions of two complex variables, $g\left(Z, Z^{*}\right)$. In analogy to the class $\mathfrak{A}\left(R_{0}\right)$ we introduce

(5) This result is a direct application of the representation of integrals of algebraic functions by $\theta$-functions and integrals of the first kind.

(6) Another direction is the study of differential equations with singular coefficients $[7 ; 9]$, and quasi-linear equations. 
certain subclasses $\mathfrak{A}\left(a_{1}, \cdots, a_{p} ; N\right)$, see p. 18, of multi-valued harmonic functions. We show that $\psi(X) \in \mathfrak{X}\left(a_{1}, \cdots, a_{p} ; N\right)$ can be represented in closed form by algebro-logarithmic expressions, $\theta$-functions, their derivatives, and finitely many transcendental functions belonging to $\mathfrak{A}\left(a_{1}, \cdots, a_{p} ; N\right)$. In $\$ \S 5$ and 6 we show that subsequences of the coefficients of their series development at the origin and at infinity belong to certain manifolds of the coefficient space.

As in the two-dimensional case, these results can be extended to the case of solutions of equation (1.3). See \$7. In particular, we obtain a representation of solutions $\psi \in \mathfrak{A}\left(a_{1}, \cdots, a_{p} ; N\right)$ of (1.3) in the form of double integrals of products of two terms. The first term depends only upon $F$ (i.e., the equation (1.3)) and the second is independent of $F$ and can be represented in a closed form, in certain cases, by the inverse of the Weierstrass 8 -function. Its argument and parameters are conveniently chosen combinations of the variables. Further we show that a subsequence of the coefficients of the series development of $\psi(\boldsymbol{X}) \in \mathfrak{H}\left(a_{1}, \cdots, a_{p} ; N\right)$ lies in a certain manifold of the coefficient space, which manifold is independent of $F$. Finally we mention the possibilities of extending our methods to more general differential equations in three variables $(\$ 8)$.

We see that due to the particular simple laws of mappings $\left({ }^{7}\right)$ by the integral operators considered a large variety of results in the theory of analytic functions may be used to obtain theorems on solutions of differential equations (1.1) and (1.3). (The theorems $2.2,7.1,7.2$, etc., are only examples in this direction.) The main interest of our considerations consists of working out a method which permits us to extend certain directions in the theory of functions, namely those dealing with the coefficient problem and classification of certain types of solutions, to the theory of equations (1.1) and (1.3).

The author wishes to thank Dr. Henry Pollak for his helpful advice and aid in the preparation of the present paper, which in some instances led to a simplification of the presentation.

2. The associate of a solution $\psi$ of equation (1.1). Solutions whose associates of the first kind are algebraic. Let $H\left(x_{1}, x_{2}\right)=\psi\left(z, z^{*}\right)$ be a (real) harmonic function of two variables; then it can be represented in the form $\left({ }^{8}\right)$

$\left({ }^{7}\right)$ The mappings $\mathbf{P}$ of one class of functions, say analytic functions $f$, onto another class, say solutions $\psi$ of (1.3), are defined at first in the small. Since $f$ and $\psi$ can be continued analytically, one can extend the definition of the mappings to the large. Since the Riemann surfaces of the corresponding functions $f$ and $\psi$ do not need to coincide, we must in the latter case indicate the sheet on which the reference point has been chosen, as well as what branch of $\psi$ is obtained by $\mathbf{P}(f)$.

(8) We note that if $x_{1}$ and $x_{2}$ are real, then $z$ and $z^{*}$ are conjugate to each other; if $x_{1}$ and $x_{2}$ are complex, $z$ and $z^{*}$ become two independent variables.

A function $\psi(z, \bar{z})$ which for real values of the arguments, $x_{1}, x_{2}$ (or $z^{*}=\bar{z}$ conjugate to $z$ ), is real, is said to be real. In the sequel $\psi$ will denote either real or complex solution of the differential equation. 


$$
\psi\left(z, z^{*}\right)=\left[g(z)+\bar{g}\left(z^{*}\right)\right] / 2, \quad z=x_{1}+i x_{2}, \quad z^{*}=x_{1}-i x_{2},
$$

so that

$$
g(z)=2 \psi(z, 0)+\text { const., } \bar{g}\left(z^{*}\right)=2 \psi\left(0, z^{*}\right)+\text { const. }
$$

Therefore the analytic function $g$, whose real part is $\psi$, can be obtained by continuing $\psi$ to complex values of the arguments and considering it in the characteristic plane $z^{*}=0$ (or $z=0$ ). In the following, we shall refer to $g$ as the associate of the first kind of $\psi$ with reference to the point $\left({ }^{9}\right) O=(0,0)$.

The Weierstrass results on integrals of algebraic functions can be interpreted in a form stating that if in the characteristic plane a harmonic function is an integral of an algebraic function, then it can be represented in the large by employing the Weierstrass formulas. Obviously various results of this kind about analytic functions can be stated for harmonic functions.

Various operators have been introduced transforming analytic functions of a complex variable into solutions of linear partial differential equations of elliptic type

$$
\begin{aligned}
L(\psi) & \equiv 4 \psi_{z z^{*}}+A \psi_{z}+B \psi_{z^{*}}+4 F \psi \\
& =\Delta \psi+\frac{1}{2}(A+B) \psi_{x}+\frac{1}{2 i}(A-B) \psi_{y}+4 F \psi=0 .
\end{aligned}
$$

In the present section, we shall show that if $A=B=0$ and $F$ is an entire function of $z, z^{*}$, the above results in the theory of functions of a complex variable can be used to derive similar results for solutions of $(1.1)\left({ }^{10}\right)$. Our considerations are based on the following theorem derived in [2] concerning the representation of solutions of the differential equation (1.1):

ThEOREM 2.1. Let $E$ satisfy the differential equation

$$
\left(1-t^{2}\right) E_{z^{*} t}-(1 / t) E_{z^{*}}+2 z t\left(E_{z z^{*}}+F E\right)=0,
$$

and certain additional conditions, then

$$
\psi=\mathbf{P}_{2}(f) \equiv \int_{t=-1}^{1} E\left(z, z^{*}, t\right) f\left(\frac{1}{2} z\left(1-t^{2}\right)\right) d t /\left(1-t^{2}\right)^{1 / 2},
$$

where $f$ is an arbitrary function regular at the origin of one complex variable, will be a (complex) solution of (1.1).

(9) Unless stated to the contrary, we shall assume, in the following, that the associate of the first kind is taken with reference to the origin, $O$. While in the case of harmonic functions in two variables, the change of the reference point does not change the character of the associate, the situation is different in the case of differential equations (2.3) and in three variables. One of the purposes of our considerations is to study solutions whose associates with respect to a conveniently chosen reference point become particularly simple.

(10) The assumption $A=B=0$ was made here only to avoid long formulas. The procedure can be repeated with almost no changes if $A$ and $B$ do not vanish. 
In [2], it has been shown that for every equation (1.1) there exist various functions $E$ of the above kind, which are defined as generating functions for an integral operator $\mathbf{P}_{2}$. In particular, there exists the function $E_{1}\left(z, z^{*}, t\right)$ having the property $E_{1}(z, 0, t)=E_{1}\left(0, z^{*}, t\right)=1$, defined as the generating function of the first kind with the reference point $O$.

If $E$ can be written in the form $1+\sum_{n=1}^{\infty} t^{2 n} Q^{(n)}\left(z, z^{*}\right)$, then the operator (2.5) can be modified. We can, namely, write instead of (2.5),

$$
\psi=\mathrm{p}_{2}(g) \equiv g(z)+\sum_{n=1}^{\infty} 2^{-n} Q^{(n)}\left(z, z^{*}\right)[\mathrm{B}(n, n+1)]^{-1} g_{n}(z)
$$

where

$$
\begin{aligned}
g & =\int_{-1}^{1} f\left(\frac{1}{2} z\left(1-t^{2}\right)\right)\left(1-t^{2}\right)^{-1 / 2} d t \\
g_{n}(z) & =\int_{0}^{z}(z-\zeta)^{n-1} g(\zeta) d \zeta \\
f(z) & =\int_{-1}^{1} g\left(z\left(1-t^{2}\right)\right) d t / t^{2}
\end{aligned}
$$

In the case of integral operators of the first kind, $Q^{(n)}(z, 0)=Q^{(n)}\left(0, z^{*}\right)=0$, $n \geqq 1$. In analogy to the situation in the case of harmonic functions, we obtain for integral operators of the first kind, the same relations (2.2) between a (real) solution $\psi\left(z, z^{*}\right)$ of (1.1) and the corresponding associate $g$ of the first kind.

REMARK 2.1. Studying complex solutions $\psi\left(z, z^{*}\right), z=x_{1}+i x_{2}, z^{*}=x_{1}-i x_{2}$, of (1.1) it is useful to distinguish a special subclass, $\mathfrak{a}$, of solutions, namely those which (when continued to complex values of the arguments) have the property that in the characteristic plane $z=0$ they become constant. In the case of equation (1.1) solutions generated by the integral operator of the first kind form the class $\mathfrak{a}$. In the special case $F=0, \mathfrak{a}$ is the class of analytic functions of a complex variable.

The aim of the present section is to characterize the class of real solutions of (1.1) which have the property that in the characteristic plane $z^{*}=0$, they become algebraic functions. (In our terminology, we shall say that the associate of the first kind of $\psi$ is an algebraic function. See also footnote 9.) In the case where $F=0$ (i.e., in the case of Laplace's equation) the harmonic function with an algebraic associate is an algebraic function of $z, z^{*}$, which is defined on a certain Riemann surface, say $\mathcal{R}_{0}$. The theory of integral operators permits us to characterize the function $\psi\left(z, z^{*}\right)$ in the case where $F$ is an entire function of $z, z^{*}$.

Formulas (2.5) and (2.6) yield a representation of these functions in the large. Thus, the solution $\psi$ of this kind can be written either in the form (2.5) where $f$ is an integral of an algebraic function of one complex variable, or in 
the form of the series (2.6) where each $g_{n}$ is an integral of an algebraic function. In particular, if we consider the class of solutions whose associates are algebraic functions defined on the given Riemann surface $R_{0}$, then the corresponding functions $f$ and $g_{n}$ can be written in a closed form using certain $\theta$-functions, their derivatives, and finitely many transcendental functions, which depend only on $R_{0}$. We proceed now to a more detailed formulation.

According to the classical results, with every closed Riemann surface $R_{0}$ defined by the irreducible equation

$$
A(z, y)=A_{0}(z) y^{n}+A_{1}(z) y^{n-1}+\cdots+A_{n}(z)=0,
$$

where $A_{\nu}(z)$, are polynomials, we can associate certain $\theta$-functions, and finitely many transcendental functions $J(z, y)_{\alpha}$. (See $[19$, pp. 516,533$]\left({ }^{11}\right)$.)

Definition 2.1. Suppose a function $N(z)$ can be represented as a finite expression involving: (1) Theta functions $\theta\left(u_{1}, \cdots, u_{\rho}\right)$, associated( $\left({ }^{12}\right)$ with $R_{0}$; (2) their derivatives with respect to the $u_{\alpha}$; (3) integrals of the first kind $J(z, y)_{\alpha}, \alpha=1,2, \cdots, \rho$, defined on $R_{0}$; (4) finitely many algebrologarithmic expressions. Then $N$ will be said to belong to the class $\mathbb{B}\left(\mathbb{R}_{0}\right)$ : $N \in \leftrightarrow\left(R_{0}\right)$.

Let $R_{1}(z)$, a new surface depending on $R_{0}$ with branch points depending on a parameter $z$, be defined by

$$
A(t, \eta ; z)=A_{0}\left(z\left(1-t^{2}\right)\right) \eta^{n}+A_{1}\left(z\left(1-t^{2}\right)\right) \eta^{n-1}+A_{n}\left(z\left(1-t^{2}\right)\right)=0 .
$$

In general, $R_{1}(z)$ has twice as many branch points as $R_{0}$. The corresponding $\theta$-functions, integrals of the first, second, and third kind [19, chaps. 12, 18, $20,30]$, and their periods, will then be functions of the parameter $z$.

Definition 2.2. Suppose a function $N_{1}(t, z)$ can be represented as a finite expression involving: (1) Theta functions $\theta\left(u_{1}, \cdots, u_{2 \sigma} \mid z\right)$ associated with $R_{1}(z) ;(2)$ their derivatives with respect to the $u_{\alpha}$; (3) integrals of the first kind $J(t, \eta \mid z)_{\alpha}, \alpha=1,2, \cdots, 2 \sigma$, defined on $R_{\mathrm{d}}(z)$; (4) finitely many algebrologarithmic expressions. Then $N_{1}$ will be said to belong to the class $\mathfrak{S}\left(\mathcal{R}_{1}\right)$ : $N_{1} \in \mathfrak{S}\left(R_{1}\right)$.

Definition 2.3. A solution $\psi\left(z, z^{*}\right)$ of (1.1) of the class $\mathfrak{a}$ which is regular at the origin and becomes in the characteristic plane $z=0$ an algebraic function, defined on the Riemann surface $R_{0}$, will be said to belong to the class $\mathfrak{A}\left(\mathbb{R}_{0}\right), \psi \in \mathfrak{A}\left(\mathbb{R}_{0}\right)$.

TheOREm 2.2. Let $\psi \in \mathfrak{A}\left(\mathbb{R}_{0}\right)$. Then the functions $g_{n}, n=1,2, \ldots$ (in the formula (2.6a)), belong to the class $\mathfrak{S}\left(\mathbb{R}_{0}\right)$, while $f$ (see (2.7)) belongs to $\mathfrak{S}\left(\mathbb{R}_{1}\right)$.

REMARK 2.2. We note that $f$ and the $g_{n}$ are independent of $F$ (see (1.1)),

(11) Here we follow as much as possible the Weierstrass original notation. It requires the use of comparatively involved formulas, but gives the possibility of seeing how the integrals depend upon parameters.

(12) $\rho$ is the genus of $R_{0}$. 
while $E$ and the $Q^{(n)}$ depend only on $F$, but not on the associate.

Proof. Our results follow directly from the fact that in the case of the integral operator of the first kind, the relation (2.2) is valid, and that $f$ and $g_{n}$ can be represented in the form (2.7) and (2.6b), respectively.

According to Weierstrass, see $\left[19\right.$, p. 264], to every Riemann surface $R_{0}$ of algebraic functions defined by $\left({ }^{13}\right) A(z, y)=0$ (see (2.8)), there exist finitely many transcendental functions (integrals of the first kind) $J(z, y)_{\alpha}$ so that the integral of the function $h(z)=R(z, y)$ (where $R$ is a rational function in $z$ and $y$ ) can be represented in the form

$$
\begin{aligned}
\int_{\left(0, y_{0}\right)}^{(z, y)} h(\zeta) d \zeta= & \sum_{\nu=1}^{r} c_{\nu} \Omega\left(z_{\nu}, y_{\nu} ; z, y ; 0, y_{0}\right) \\
& +\sum_{\alpha=1}^{\rho}\left[h_{\alpha}^{\prime} J(z, y)_{\alpha}-h_{\alpha} J^{\prime}(z, y)_{\alpha}\right]+\sum_{\nu=1}^{r} H_{\nu},
\end{aligned}
$$

where $r$ is the number of simple poles of $h, \rho$ is the genus, and the $H_{\nu}$ are algebraic functions (see [19, pp. 264, 382, and 373]). According to [19, pp. 383, 596],

$$
\begin{aligned}
\Omega\left(z_{v}, y_{v} ; z, y, 0, y_{0}\right)=\Omega\left(z_{0}, y_{0} ; z, y, 0, y_{0}\right) \\
+\log \left(\frac{\theta\left(w-w_{1}-\tilde{w}_{1}, \cdots\right) \theta\left(w^{\prime}-\tilde{w}_{1}-\tilde{w}_{1}, \cdots\right)}{\theta\left(w-w_{1}-\tilde{w}_{1}, \cdots\right) \theta\left(w^{\prime}-w_{1}-\tilde{w}_{1}, \cdots\right)}\right) \\
\quad+\sum_{\alpha=1}^{\rho}\left(\begin{array}{c}
\alpha \\
w \\
w^{\prime}
\end{array}\right)\left\{J^{\prime}(z, y)_{\alpha}-J^{\prime}\left(z_{0}, y_{0}\right)_{\alpha}\right\} . \\
J^{\prime}(z, y)_{\alpha}=\frac{\stackrel{(\alpha)}{\theta}\left(w_{1}-\tilde{w}_{1}-w_{1 \alpha}, \cdots\right)}{\theta\left(w_{1}-\tilde{w}_{1}-w_{1 \alpha}, \cdots\right)}
\end{aligned}
$$

Here $\quad \stackrel{(\alpha)}{\theta}=\partial \theta / \partial u_{\alpha}, \quad w_{\alpha \beta}=J\left(a_{\alpha}, b_{\alpha}\right)_{\beta}$,

$$
\begin{aligned}
J\left(z_{1}, y_{1}\right)_{\alpha} & =\stackrel{\alpha}{w}, \quad J\left(z_{1}^{\prime}, y_{1}^{\prime}\right)=\stackrel{\alpha}{w^{\prime},} & & {[19, \text { p. 597], }} \\
\tilde{w}_{\alpha} & =\frac{1}{2} \sum_{\nu=1}^{2 \rho-2} J\left(p_{\nu}, q_{v}\right), & & {[19, \text { p. 593], }} \\
J\left(0, y_{0}\right)_{\beta} & =\stackrel{0}{w}_{\beta}, & & {[19, \text { p. 486], }}
\end{aligned}
$$
$\operatorname{Im} z$.

(13) Here $y$ is an algebraic function of $z$ defined by (2.8). (It should not be confused with 
are constants, and

$$
w_{\beta}=J(z, y)_{\beta}
$$

integrals of the first kind.

We proceed now with the proper proof of Theorem 2.2. We note that if we write $\int_{\left(0, y_{0}\right)}^{(z, y)} g(\zeta) d \zeta$ in the canonical form $(2.10)$, i.e.,

$$
\begin{aligned}
\int_{\left(0, y_{0}\right)}^{(z, y)} g(\zeta) d \zeta & =\sum_{\nu=1}^{r} c_{\nu} \Omega\left(z_{\nu}, y_{\nu} ; z, y ; 0, y_{0}\right)-\sum_{\alpha=1}^{\rho}\left(g_{\alpha}^{\prime} J(z, y)_{\alpha}-g_{\alpha} J^{\prime}(z, y)_{\alpha}\right)+\sum_{\nu=1}^{r} G_{\nu}, \\
g_{\alpha} & =\sum_{\nu=1}^{r} G_{\nu, \alpha}, g_{\alpha}^{\prime}=\sum_{\nu=1}^{r} G_{\nu, \alpha}^{\prime}-c^{(\alpha)}
\end{aligned}
$$

$[19$, p. 264$]$, then

$$
\begin{aligned}
\int_{\left(0, y_{0}\right)}^{(z, y)} \zeta^{\lambda} g(\zeta) d \zeta & =\sum_{\nu=1}^{r} a_{\nu}^{\lambda} F_{\lambda}, & & \lambda \text { integer } \geqq 1, \\
\int_{\left(0, y_{0}\right)}^{(z, y)}(z-\zeta)^{n} g(\zeta) d \zeta & =\sum_{\nu=1}^{r}\left(z-a_{\nu}\right)^{n} F_{\nu}, & & n \text { integer } \geqq 1,
\end{aligned}
$$

where

$$
\begin{aligned}
F_{\nu}=\left[c _ { \nu } \Omega \left(z_{\nu}, y_{\nu} ; z, y\right.\right. & \left.; 0, y_{0}\right) \\
& \left.-\left(\sum_{\alpha=1}^{\rho}\left(G_{\nu, \alpha}^{\prime}-c^{(\alpha)}\right) J(z, y)_{\alpha}-G_{\nu, \alpha} J^{\prime}(z, y)_{\alpha}\right)+G(z, y)_{\nu}\right] .
\end{aligned}
$$

Here $a_{v}$ represent the poles of $g(\zeta)$.

REMARK 2.3. Concerning the determination of the value $r$ : If $g(\zeta)$ has a branch point at infinity, $\zeta^{\lambda} g(\zeta)$ will also have a branch point of the same order at infinity. If, however, $g$ is regular at infinity, or has a pole there, then $\zeta^{\lambda} g(\zeta)$ may have, for one value of $\lambda$, a simple pole at infinity. Thus it is necessary, in order to make our formula valid for all $\lambda$, to include among the simple poles of $g$ the point at infinity; and we shall have to introduce a function of the third kind with pole there. The corresponding coefficient $c_{\nu}$ can then be nonzero for only one value of $\lambda$.

The first part of Theorem 2.2 now follows immediately from (2.6b) and Definition 2.1.

To prove the second part of Theorem 2.2, namely the representation of $f(z)$, we only need to recall formula (2.7). It is then obvious that $f \in S_{C}\left(R_{1}\right)$.

In the next section, we shall describe in more detail the Riemann surfaces on which a solution $\psi$ with an algebraic associate is defined.

3. The Riemann surface of a solution $\psi$ of (1.1) with an algebraic associate. The representation (2.6), (2.6a), and (2.6b) allows us to describe the 
Riemann surface $R$ on which $\psi$ is single-valued.

Suppose $R_{0}$ is the Riemann surface defined by equation (2.9) whose genus is $\rho>0$. As shown in [4, p. 318], every branch point $Q$ of finite order of $g$ is transformed by the operator $\mathbf{p}_{2}(g)$, see (2.6), into a branch point of the same order, and at the same point $Q$, while every pole of $g$ is transformed into a singularity of $\mathbf{p}_{2}(g)$ at the same point, but at which $\mathbf{p}_{2}(g)$ has a branch point of infinite order $\left({ }^{14}\right)$.

Let $\widetilde{R}=\sum R_{0}^{(\nu)}, \nu=1,2, \cdots$, be the universal covering surface of $R_{0}$. Further, let $P_{1}, P_{2}, \cdots, P_{n}$ be the poles of $g$ on $R_{0}$, and let there correspond, to the points $P_{1}, P_{2}, \cdots, P_{n}$, on every copy $R_{0}^{(\nu)}$, the points $P_{1}^{(\nu)}, P_{2}^{(\nu)}, \cdots$, $P_{n}^{(\nu)}$. Since at every point $\Phi_{k}^{(\nu)}$ is located a branch point of infinite order, we must, at every point $\mathscr{P}_{\kappa}^{(\nu)}$, attach $\left.{ }^{15}\right)$ infinitely many copies $\widetilde{R}_{\nu, k}$ of $\widetilde{R}$. $R$ $=\sum_{k=-\infty}^{\infty} \sum_{\nu=-\infty}^{\infty} R_{\nu, \kappa}$ is the Riemann surface on which $\psi=\mathbf{p}_{2}(g)$ is singlevalued.

REMARK 3.1. The above considerations allow the conclusion that there exist, in general, Riemann surfaces on which no solutions of the differential equation (1.1), with $F \neq 0$, can be single-valued. For instance, suppose $S$ is a closed Riemann surface of the function

$$
y=\left[\left(z-a_{1}\right)\left(z-a_{2}\right)\left(z-a_{3}\right) \cdots\left(z-a_{2 n}\right)\right]^{1 / 2}, \quad n>1 .
$$

Suppose, further, that $\psi(z, \bar{z}), \bar{z}$ conjugate to $z$, is a (real) solution of (1.1) whose Riemann surface is $\left(^{16}\right) \delta$. If we continue the values of the arguments $x_{1}$ and $x_{2}$ of $\psi\left(x_{1}+i x_{2}, x_{1}-i x_{2}\right)$ to complex values (i.e., assume that $z$ and $z^{*}$ are not necessarily conjugate to each other), then by continuing to the complex values of $x_{1}$ and $x_{2}$, we obtain the function $\psi\left(z, z^{*}\right)$ defined for $|z|<\infty$, $\left|z^{*}\right|<\infty . \psi\left(z, z^{*}\right)$ possesses, as the only singularities, branch planes $z=a_{v}$ and $z^{*}=\bar{a}_{\nu}, \nu=1,2, \cdots, 2 n$.

In the characteristic plane $z^{*}=0$ (or $z=0$ ), the function $\psi(z, 0)$ is regular everywhere except at the points in which they intersect the branch planes of $\psi\left(z, z^{*}\right)$, i.e., in points $a_{1}, a_{2}, \cdots, a_{2 n}$. It could happen, however, that the value of the function $\psi(z, 0)$ in two different sheets in the neighborhood of a point $a_{\nu}$ coincide, so that we have no branch point at all. Thus, we have to consider different possibilities. At first, let us suppose that $\psi(z, 0)$ has branch points of the first order at the points $a_{1}, a_{2}, \cdots, a_{2 n}$. Then, according to our previous considerations, the corresponding function must be infinitely many-valued, since $n>1$, and $\psi(z, \bar{z}), \bar{z}$ conjugate to $z$, is not single-valued on $\delta$.

If some point $a_{\nu}$ is a regular point of $\psi(z, 0)$, instead of a branch point,

(14) Thus, the location and character of these singularities of $\mathrm{p}_{2}(g)$ are independent of $F$.

(15) Cutting $R$, we have to connect $\mathscr{P}_{\kappa}^{(\nu)}$ with $\infty$ in such a way that the cuts have no intersections with each other.

${ }^{(16)}$ This means, in particular, that $\psi(z, \bar{z})$ has as the only singularities branch points of the second order at $a_{\nu}$ and $\bar{a}_{\nu}, \nu=1,2, \cdots, 2 n$. 
then $\psi(z, \bar{z})$ is regular at $a_{\nu}$ which again contradicts the fact that $\psi(z, \bar{z})$ should have a branch point of first order at $a_{\nu}$. Therefore (if the $Q^{(n)}$ are not identically equal to zero) we have a contradiction in both cases, which shows that the surface $S$ cannot be the Riemann surface of a solution $\psi(z, \bar{z})$.

The coefficients of $g$ coincide with the subsequence $\left\{a_{m 0}\right\}$ of the development $\psi(z, \bar{z})=\sum a_{m n} z^{m} \bar{z}^{n}$ of a real solution. Thus, using the classical results on functions of one complex variable, we can describe properties of the coefficients $a_{m 0}$ of the function element $\sum_{m=0}^{\infty} a_{m 0} z^{m}$ of $g$. In particular, if $g$ is an algebraic function, then the coefficients $\left\{a_{n 0}\right\}$ will satisfy the Eisenstein conditions (see $[10$, p. 332]). In addition to that we can easily formulate conditions that $g$ be defined on a given Riemann surface, $R_{0}$, and therefore $\psi=\mathbf{p}_{2}(g) \in \mathfrak{A}\left(\boldsymbol{R}_{0}\right)$ and is defined on the corresponding $R$. It is of interest that these conditions are independent of $F$. For the sake of simplicity we discuss these conditions only when $g$ is a two-valued algebraic function. In this case $g$ may be written in the form

$$
g(z)=\frac{Q(z)}{(R(z))^{1 / 2}}=\sum_{\nu=0}^{N} \epsilon_{\nu} z^{\nu} /\left(\prod_{1}^{p}\left(z-a_{i}\right)\right)^{1 / 2} .
$$

Let

$$
\sum_{n=0}^{\infty} \gamma_{n} z^{n}
$$

be the series development of $g$. Then since all coefficients $\gamma_{n}$ depend linearly upon finitely many $\epsilon_{\nu}, \nu=0,1, \cdots, N$, one can determine a set of numbers $\delta_{n}$, $n=1,2, \cdots$, depending only on the $a_{j}$, such that in

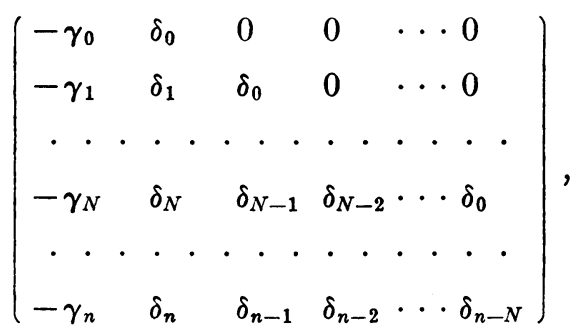

every subdeterminant of order $N+2$ vanishes. Since (as it is possible to show) none of these relations vanishes identically, we obtain infinitely many linear relations for the $\gamma_{\nu}$.

If $\psi=\operatorname{Re} \mathbf{p}_{2}(g)$, and $\psi=\sum a_{m n} z^{m} z^{* n}$, then $a_{m 0}=\gamma_{m}$ and therefore the same equations hold for $\left\{a_{m 0}\right\}$. These conditions in order that $\psi$ belong to the class of multivalued solutions described in $\$ 2$ are independent of $F$.

In $\S 7$ we shall show that results of this kind hold also for equations (1.3) in three variables.

4. Harmonic functions with an algebraic $C_{3}$ associate of a certain form. 
In $\S \S 2$ and 3 using the method of integral operators we studied properties of solutions of differential equations in two variables.

In the following we shall show that at least for some classes of differential equations in three variables, namely of the form (1.3), analogous results can be obtained. As we stressed in the introduction, the major task arising in our study of differential equations of the form (1.3) is the investigation of the mappings indicated in $\$ 1$ onto harmonic functions $H(X)$ of three variables. Compared with the situation in the two-dimensional case, this study is much more involved for the following reasons:

(1) The associate functions which arise when we consider $H(X)$ in the characteristic space by substituting

$$
x_{1}=2\left(Z Z^{*}\right)^{1 / 2}, \quad x_{2}=-i\left(Z+Z^{*}\right), \quad x_{3}=\left(Z-Z^{*}\right)
$$

are functions of two complex variables, and this theory is not yet developed as far as that of one variable.

(2) The simplest representation (now known) for an integral operator transforming functions of two complex variables $\chi\left(Z, Z^{*}\right)=\chi_{1}\left(Z, Z^{*}\right)$ $+\left(Z Z^{*}\right)^{1 / 2} \chi_{2}\left(Z, Z^{*}\right) \equiv H\left(2\left(Z Z^{*}\right)^{1 / 2},-i\left(Z+Z^{*}\right),\left(Z-Z^{*}\right)\right)$ into corresponding harmonic functions, regular at the origin,

$$
\begin{aligned}
H(X)=\mathrm{C}_{3}(\chi) & \equiv \frac{1}{\pi i} \int_{|\zeta|=1}\left[\int_{T=0}^{1} u^{1 / 2} \frac{d \chi\left(u \zeta^{-1} T^{2}, u \zeta(1-T)^{2}\right)}{d u} d T\right] \frac{d \zeta}{\zeta} \\
u & =x_{1}+2^{-1}\left(i x_{2}+x_{3}\right) \zeta+2^{-1}\left(i x_{2}-x_{3}\right) \zeta^{-1}
\end{aligned}
$$

involves two integrations. See $[8$, p. 468].

Here the $\chi_{k}, k=1,2$, are functions which are regular at the origin.

(3) Singularities of harmonic functions of three variables when considered in the real space may degenerate; for instance, the line singularities become, in some exceptional cases, points.

These complications suggest that in addition to the mapping of the space of harmonic functions onto the algebra of the functions in the characteristic space, we investigate mappings onto other algebras. In particular, those mappings where the transition from the associates to the harmonic functions is achieved by one integration are of considerable interest, since in this case we may exploit classical results on integrals of functions of one complex variable belonging to certain classes.

Two such mappings have been extensively studied.

1. The mappings of the rational and algebraic $\mathbf{B}_{3}$-associates $f$, into harmonic functions

$$
H(\boldsymbol{X})=\mathbf{B}_{3}(f) \equiv \int_{\mathcal{L}^{1}} f(u, \zeta) d \zeta / \zeta, \quad \boldsymbol{X} \in \mathcal{V}^{3}\left(\boldsymbol{X}_{1}\right)
$$


2. The mappings of the ring of polynomials $Q(\zeta)$ into harmonic functions

$$
H(\boldsymbol{X})=\int_{\mathcal{L}^{1}} \cdot \frac{Q(\zeta) d \zeta}{(P(\zeta, \boldsymbol{X}))^{1 / 2}}, \quad \boldsymbol{X} \in \mathcal{V}^{3}\left(\boldsymbol{X}_{1}\right)
$$

see [5], where

$$
\begin{aligned}
P(\zeta, X) & =\sum_{k=1}^{3}\left(x_{k}-u_{k}(\zeta)\right)^{2}=\rho^{2}+R^{2}(\zeta)-2 R(\zeta) \rho T \\
T & =\cos \theta \cos \theta_{1}(\zeta)+\sin \theta \sin \theta_{1}(\zeta) \cos \left(\phi_{1}(\zeta)-\phi\right) \\
\rho^{2} & =x_{1}^{2}+x_{2}^{2}+x_{3}^{2} .
\end{aligned}
$$

$U_{3}\left(X_{1}\right)$ represents a sufficiently small neighborhood of the point $X_{1} \cdot \mathcal{L}^{1}$ is a smooth, open curve in the $\zeta$-plane with the end points $\zeta_{1}$ and $\zeta_{2}$ such that $\left({ }^{17}\right)$

$$
\mathcal{L}^{1} \cap\left[P(\zeta, X)=0, X \in \mho^{3}\left(X_{1}\right)\right]=0 .
$$

In cases (1) and (2) we obtained harmonic functions possessing singularities on algebraic curves. The results obtained for harmonic functions $H(X)$ were employed to obtain solutions of (1.3) possessing singularities on algebraic curves. In (4.9) of [8] we introduced the operator $\mathrm{p}_{3}(G)$ (transforming the harmonic functions $G$ into solutions of (1.3)) in the form

$$
\mathrm{p}_{3}(G)=G(X)+2 \sum_{n=1}^{\infty} \frac{\Gamma(n+1 / 2)}{\Gamma(1 / 2) \Gamma(n)} b^{(n)}\left(r^{2}\right) \int_{\sigma=0}^{1}\left(1-\sigma^{2}\right)^{n-1} \sigma^{2} G\left(\sigma^{2} X\right) d \sigma .
$$

This formula permits us to obtain in the three-dimensional case results which are analogous to those which we discussed in $\$ \S 2$ and 3 for the twodimensional case. In particular, if $G(\boldsymbol{X})$ is an algebraic function,

$$
\int_{\sigma=0}^{1}\left(1-\sigma^{2}\right)^{n-1} \sigma^{2} G\left(\sigma^{2} X\right) d \sigma, \quad n=1,2, \cdots,
$$

will be integrals of algebraic functions and therefore according to the classical results can be represented by certain $\theta$-functions, their derivatives, and finitely many transcendental functions. See for details p. 506 of [8]. In a similar way the formula (4.6) permits us to exploit other results on integrals of algebraic functions in the theory of differential equations in three variables. In this connection it is of importance to characterize the $\mathbf{C}_{3}$-associates of the harmonic functions which have been introduced and investigated in cases (1) and (2).

In case (1) it was possible to characterize the harmonic functions (4.3) where $\mathcal{L}^{1}$ is a closed curve. In this case, provided that $f$ (i.e., the $\mathbf{B}_{3}$-associates) are algebraic, the $\mathbf{C}_{3}$-associates are combinations of algebro-logarithmic

(17) In case (1), $\zeta_{1}$ and $\zeta_{2}$ usually coincide, so that $\mathcal{L}^{1}$ is a closed curve. 
functions and so-called period functions. It was not possible in the general case to characterize in a simple way the $\mathbf{C}_{3}$-associates corresponding to case (2). As we shall show, however, in $\$ 5$, there exists an interesting subclass of these functions (which will be denoted by 5 ) whose $\mathbf{C}_{3}$-associates are functions of a certain form of one variable. This variable is a conveniently chosen combination of the variables $Z$ and $Z^{*}$. Using these properties, as we shall indicate in $\S \S 5$ and 6 , it is possible to characterize to a large extent the harmonic functions of the subclass $\mathfrak{S}$.

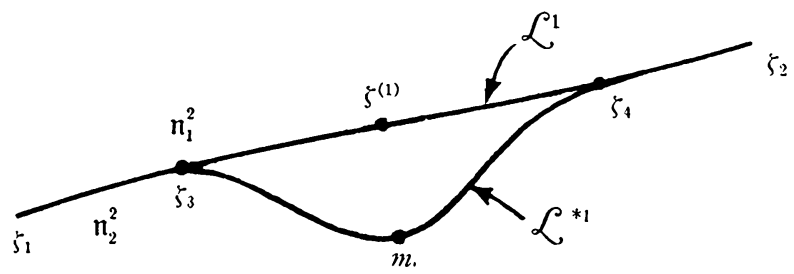

FIG. 1

Before proceeding to this more special consideration, it will be useful (1) to make a table of the operators in the present paper, (2) to prove in Lemma 4.1 certain results concerning the analytic continuation of harmonic functions given by (4.4) provided that $Q(\zeta)$ is a polynomial.

$\mathbf{p}_{2}, \quad$ (2.6), transforms analytic functions $g(z)$ into solutions of (1.1) of the class $\mathfrak{a}$, see Remark 2.1 , and is connected with

$\mathrm{P}_{2}, \quad$ (2.5), by relation (2.7).

$\mathrm{C}_{3}$, (4.2), transforms functions $\chi\left(Z, Z^{*}\right)$ of two complex variables $Z, Z^{*}$ into (complex) harmonic functions of three variables.

$\mathbf{B}_{3}, \quad$ (4.3), transforms analytic functions of

$$
u=\left(x_{1}+2^{-1}\left(i x_{2}+x_{3}\right) \zeta+2^{-1}\left(i x_{2}-x_{3}\right) \zeta^{-1}\right) \text { and } \zeta
$$

into (complex) harmonic functions of $X=\left(x_{1}, x_{2}, x_{3}\right)$.

$\mathbf{p}_{3}$, (7.1), transforms complex harmonic functions of three variables into complex solutions of (1.3), connected with

$\mathrm{P}_{3}, \cdot(7.1)$, by relation (7.1a).

Lemma 4.1. $H(X)$ defined by (4.4) can be continued analytically to all finite points except

$$
\dot{s}_{1}^{1}=\left[P(\zeta, X)=0, P_{\zeta}(\zeta, X)=0\right] \text { and } \dot{B}_{2}^{1}=\left[P\left(\zeta_{k}, X\right)=0, k=1,2\right] .
$$

Proof. If $P\left(\zeta, X_{0}\right) \neq 0$ for all $\zeta \in \mathcal{L}^{1}$, then (4.4) represents a harmonic function for $\boldsymbol{X} \in \mathcal{U}^{3}\left(\boldsymbol{X}_{0}\right)$ where $\mho^{3}\left(\boldsymbol{X}_{0}\right)$ is a sufficiently small neighborhood of $X_{0}$. Let $X_{1}$ be a point where $P\left(\zeta^{(1)}, X_{1}\right)=0$ for a value $\zeta^{(1)} \in \mathcal{L}^{1}$ and let $P_{\zeta}\left(\zeta^{(1)}, X_{1}\right) \neq 0$. In order to prove our lemma we have to show that $H(X)$ 
can be continued analytically to the value $X_{1}$. This will be proved by a suitable variation of the integration curve $\mathcal{L}^{1}=\left[\zeta_{1} \zeta^{(1)} \zeta_{2}\right]$ to a new curve $\mathcal{L}^{* 1}$.

To every point $\zeta_{0}$ and the $\zeta$-plane there corresponds a circle

$$
\begin{aligned}
\mathcal{P}^{1}\left(\zeta_{0}\right) & =\left[\sum_{k=1}^{3}\left(x_{k}-v_{k}\left(\zeta_{0}\right)\right)^{2}=\sum_{k=1}^{3} w_{k}^{2}\left(\zeta_{0}\right), \sum_{k=1}^{3}\left(x_{k}-v_{k}\left(\zeta_{0}\right)\right) w_{k}\left(\zeta_{0}\right)=0\right], \\
u_{k}\left(\zeta_{0}\right) & =v_{k}\left(\zeta_{0}\right)+i w_{k}\left(\zeta_{0}\right),
\end{aligned}
$$

(which can degenerate to a point) where $P\left(\zeta_{0}, X\right)$ vanishes. To an arc

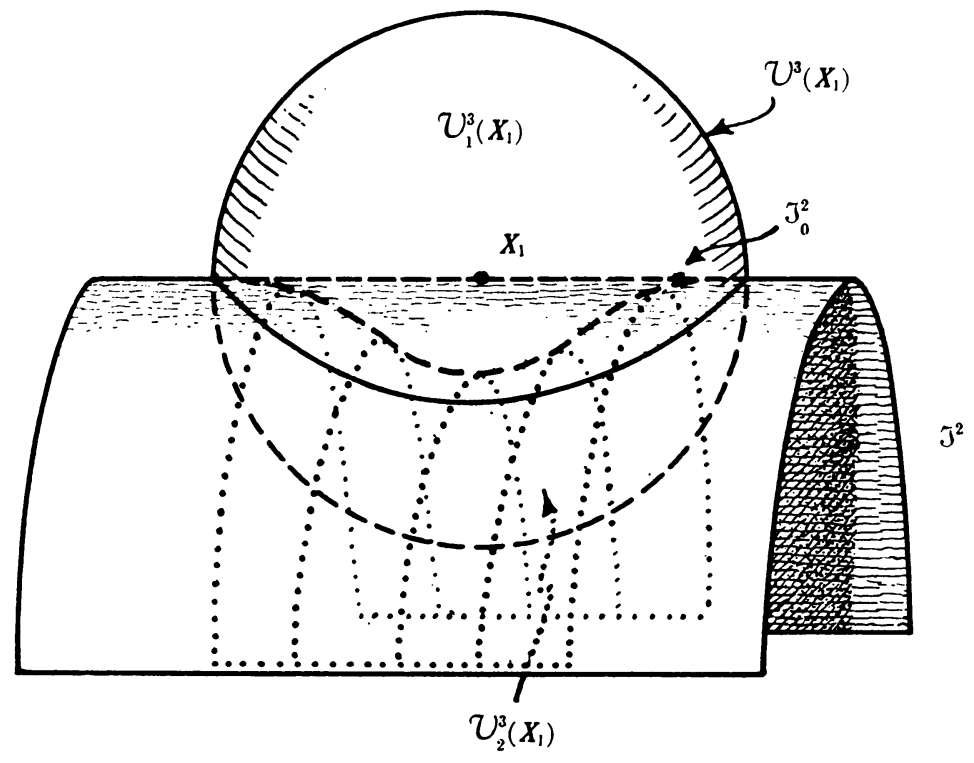

FIG. 2

$\mathcal{L}^{1}=\left[\zeta_{1} \zeta^{(1)} \zeta_{2}\right]$, see Fig. 1 , there corresponds in the $X$-space a two-dimensional manifold

$$
J^{2}=\left[\sum \mathcal{P}^{1}(\zeta), \zeta \in \mathcal{L}^{1}+\mathcal{L}^{0}\right], \mathcal{L}^{0}=\left(\zeta_{1}, \zeta_{2}\right)
$$

which has the property that if $P(\zeta, X)=0$ for $\zeta \in \mathcal{L}^{1}+\mathcal{L}^{0}$, then $X$ must belong to $J^{2}$. (Note that the set of points consisting of $\zeta_{1}$ and $\zeta_{2}$ is denoted by $\left(\zeta_{1}, \zeta_{2}\right)$.)

The values of $X$ for which $P(\zeta, X)=0$ lie on the circle $\mathcal{P}^{1}(\zeta)$. Let $P\left(\zeta^{(1)}, X\right)$ $=\prod_{k=1}^{p}\left(\zeta^{(1)}-e_{k}(X)\right)$. Suppose now that $e_{1}\left(X_{1}\right)=\zeta^{(1)}$, then $e_{k}\left(X_{1}\right) \neq \zeta^{(1)}$ for $k=2,3, \cdots, p$, since $P_{\zeta}\left(\zeta^{(1)}, X_{1}\right) \neq 0$.

Let $U^{3}\left(X_{1}\right)$ be a neighborhood of $\boldsymbol{X}_{1}$ which is so small that

1. $e_{k}(X), X \in U^{3}\left(X_{1}\right), k=1,2, \cdots, p$, is single-valued;

2. $\left|e_{k}(X)-e_{1}(X)\right|>A>0, k=2,3, \cdots, p$, where $A$ is some fixed number;

3. $\mho^{3}\left(X_{1}\right)$ is divided by $\Im^{2}$ in to two (connected) parts $\mho_{1}^{3}\left(X_{1}\right)$ and $\mho_{2}^{3}\left(X_{1}\right)$. 
Then we have:

$$
\mho^{3}\left(X_{1}\right)=\mho_{1}^{3}\left(X_{1}\right)+\vartheta_{2}^{3}\left(X_{1}\right)+\Im_{0}^{2}, \quad \Im_{0}^{2}=\Im^{2} \cap \mho^{3}\left(X_{1}\right) .
$$

Since $e_{1}(X)$, for $X \in U^{3}\left(X_{1}\right)$, is single-valued, by the relation

$$
\zeta=e_{1}(X),
$$

a one-to-one mapping of points $\zeta$ into segments of lines

$$
\dot{i}^{1}(\zeta)=\mathbb{P}^{1}(\zeta) \cap \mho^{3}\left(X_{1}\right)
$$

is defined. $J_{0}^{2}$ divides $U^{3}\left(X_{1}\right)$ into two parts. Since to the points $\zeta$ lying on $\mathcal{L}^{1}$ correspond segments $\dot{j}^{1}(\zeta)$ lying on $\Im_{0}^{2}$, the values of $\zeta=e_{1}(X), X \in V_{1}^{3}\left(X_{1}\right)$, lie on one side, say $\mathfrak{n}_{1}^{2}$; for $X \in \mathcal{V}_{2}^{3}\left(X_{1}\right)$ on the other side, $\mathfrak{n}_{2}^{2}$, of the segment $\mathcal{L}^{1}$, see Fig. 1.

Let $\mathcal{L}^{* 1}$ be a curve which connects $\zeta_{1}$ and $\zeta_{2}$, and is obtained by replacing a sufficiently small arc $\left[\zeta_{3} \zeta^{(1)} \zeta_{4}\right]$ of $\mathcal{L}^{1}$ by a circular arc, $\left[\zeta_{3} m \zeta_{4}\right]$, which lies (except the end points) in $\mathfrak{n}_{2}^{2}$.

Then for $X \in \mathcal{V}_{1}^{3}\left(X_{1}\right)$ we obtain two representations

$$
\begin{aligned}
H(\boldsymbol{X}) & =\int_{\mathcal{L}^{1}} Q(\zeta)[P(\zeta, \boldsymbol{X})]^{-1 / 2} d \zeta \\
& =\int_{\mathcal{L}^{* 1}} Q(\zeta)[P(\zeta, \boldsymbol{X})]^{-1 / 2} d \zeta .
\end{aligned}
$$

To the curve $\mathcal{L}^{* 1}$ there corresponds a segment of a new surface $J^{* 2}$, which lies in $V_{2}^{3}\left(X_{1}\right)+J_{0}^{2}$ but does not contain $X_{1}$. Hence there exists a sufficiently small neighborhood, say $\widetilde{U}^{3}\left(X_{1}\right)$, which does not intersect $\Im^{* 2}$ and in which consequently $P(\zeta, X), \zeta \in \mathcal{L}^{* 1}$, does not vanish. Hence $(4.11 \mathrm{~b})$ exists for $\boldsymbol{X} \in \tilde{\mathcal{U}}^{3}\left(\boldsymbol{X}_{1}\right)$ and represents the analytic continuation of $H(\boldsymbol{X})$ to $\tilde{\mathcal{U}}^{3}\left(\boldsymbol{X}_{1}\right)$. $\xi_{1}^{1}$ and $s_{2}^{1}$ are (one-dimensional) algebraic curves, and hence they cannot divide the (three-dimensional) $X$-space into two parts.

This completes the proof of Lemma 4.1. There now arises the question of how many independent harmonic functions $H_{\nu}(X)$ are represented by (4.4) with fixed end points $\zeta_{1}, \zeta_{2}$.

For every $X$ not belonging to $\mathbb{8}_{1}^{1}$, see $(4.7),(P(\zeta, X))^{1 / 2}$ is a function of $\zeta$ which is determined on a two-sheeted Riemann surface whose branch points $\zeta=e_{k}(X)$ vary with $X$.

Let $\mathcal{L}^{1}$ be a fxed curve on the (two-sheeted) Riemann surface of $(P(\zeta, X))^{1 / 2}$ with the end points $\zeta_{1}$ and $\zeta_{2}$.

If now we vary the point $X_{1}$, the right-hand side of (4.4) will yield the same harmonic function until one of the branch points $e_{k}\left(X_{1}\right), k=1,2, \cdots, p$, intersects $\mathcal{L}^{1}$. As we indicated before, the relation

$$
e_{k}(X)=\zeta, \quad k=1,2, \cdots, p,
$$


for a fixed $\zeta$ defines the circle $P^{1}(\zeta)$ in the $X$-space. The surface $J^{2}$, see (4.9), will (in general) divide the three-dimensional space into a number of cells, say $C_{\nu}^{3}\left(\mathcal{L}^{1}\right), \nu=1,2, \cdots$. The right-hand side of $(4.4)$ will represent in each of the cells $\mathcal{C}_{\nu}^{3}\left(\mathcal{L}^{1}\right), \nu=1,2, \cdots$, a harmonic function.

On the other hand, in different cells $\bigodot_{\nu}^{3}\left(\mathcal{L}^{1}\right)$, the right-hand side of (4.4) will represent (in general) different harmonic functions, say $H_{1}(\boldsymbol{X})$, $H_{2}(\boldsymbol{X}), \cdots$. As indicated before, each function $H_{\nu}(\boldsymbol{X}), \nu=1,2, \cdots$, can be analytically continued outside the cell $\bigodot_{\nu}^{3}\left(\mathcal{L}^{1}\right)$, by conveniently varying the curve $\left({ }^{18}\right) \mathcal{L}^{1}$.

According to Lemma 4.1 each $H_{\nu}(\boldsymbol{X})$ is defined by analytic continuation in

$$
A^{3}-\mathfrak{B}^{1}, \quad \mathfrak{B}^{1}=\mathfrak{B}_{1}^{1}+\mathfrak{B}_{2}^{1},
$$

where $A^{3}=\left[x_{1}^{2}+x_{2}^{2}+x_{3}^{2}<\infty\right]$, and $\mathbb{B}_{1}^{1}$ and $\mathbb{B}_{2}^{1}$ were introduced in (4.7).

Let us consider the (three-dimensional) domain $A^{3}-\mathfrak{g}^{1}$. Its one-dimensional Betti number will in general be different from zero. We now make the assumption that by a finite number of cuts, $\mathcal{T}_{m}^{2}, m=1,2, \cdots, n, \mathcal{T}^{2}$ $=\sum_{m=1}^{n} \mathcal{T}_{m}^{2}$, we can make the space $\mathcal{A}^{3}-\mathfrak{g}^{1}$ simply-connected. If we move along a closed curve, $\mathcal{D}^{1}$, starting from the point $X_{0}$, which curve $\mathcal{D}^{1}$ can be reduced to a point in $\mathcal{A}^{3}-\mathfrak{B}^{1}$, then obviously upon returning to the values $X_{0}$ we shall have the same integration curve $\mathcal{L}^{1}$ from which we started, and therefore we shall get for $H(\boldsymbol{X})$ the initial value $H\left(\boldsymbol{X}_{0}\right)$.

If, however, our curve cannot be reduced to a point in the space $A^{3}-\mathbb{B}^{1}$ (in which case $\mathcal{D}^{1}$ cuts the surface $\mathcal{T}^{2}$ ), then, in general, upon returning to the starting point $\boldsymbol{X}_{0}$, we shall obtain a new curve, say $\mathcal{L}_{2}^{1}$, whose end points are $\zeta_{1}$ and $\zeta_{2}$, but which cannot be deformed continuously on the Riemann surface $R\left(X_{0}\right)$ to $\mathcal{L}^{1}, R(X)$ being the Riemann surfaces over the $\zeta$-plane with branch points $e_{k}\left(X_{0}\right)$, see (4.12). Therefore, the difference between the new function, say $H^{*}\left(\boldsymbol{X}_{0}\right)$, and the function $H\left(\boldsymbol{X}_{0}\right)$ from which we started is

$$
\sum_{\nu=1}^{2 p} a_{\nu}\left(X_{0}\right) \Omega_{\nu}\left(X_{0}\right)
$$

where $\Omega_{\nu}\left(X_{0}\right)$ are so-called period functions and $a_{\nu}\left(X_{0}\right)$ are integers. The theory of period functions was developed in [1] where it was shown that our functions are defined on $R$-manifolds with infinitely many sheets and a method has been discussed, by which, using the theory of hyperelliptic integrals of one complex variable, we get some information about these functions. In particular, it has been shown that if we introduce certain $\theta$-functions and their derivatives, the totality of the functions $H(\boldsymbol{X})$ which one obtains where $Q$ ranges over the totality of polynomials has in a certain sense a finite basis: using the above mentioned $\theta$-functions, their derivatives, and finitely

(18) The end points $\zeta_{1}$ and $\zeta_{2}$ of $\mathcal{L}_{1}^{1}$ naturally have to be fixed. 
many algebro-logarithmic expressions, the $\Omega_{\nu}(X)$ can be represented by finitely many transcendental functions. The functions belonging to this basis are certain period functions mentioned above.

Thus our method permits us to generate, by evaluating the right-hand side of (4.4) and varying the neighborhood $V^{3}\left(X_{1}\right)$, a number of multivalued harmonic functions. They differ from each other by a combination (4.14) of period functions.

As indicated before, the curves $\mathbb{B}_{1}^{1}$ and $\mathbb{B}_{2}^{1}$ are the only possible singularity lines $\left({ }^{19}\right)$ of functions $H_{\nu}(\boldsymbol{X})$.

Lemma 4.2. $z_{2}^{1}=\sum_{k=1}^{2} P\left(\zeta_{k}\right)$ consists of two circles, see (4.7), in the $X$-space.

Lemma 4.3. Let $2 p_{1}$ be the degree in $\zeta$ of $P(\zeta, X)$. The curve $8_{1}^{1}$ is (in general) an algebraic curve of the degree not higher than

$$
5 p_{1}-3 .
$$

Proof. In (4.5) we expressed $P(\zeta, X)$ in polar coordinates $\rho, \theta$, and $\phi$. In order to determine the discriminant of our equation $P(\zeta, X)=0$, we write down the coefficients of $\zeta^{\nu}, \nu=0,1, \cdots$, in

$$
\begin{gathered}
P_{\zeta}, P, \zeta P_{\zeta}, \zeta P, \cdots, \zeta^{2 p_{1}-2} P_{\zeta}, \zeta^{2 p_{1}-2} P, \zeta^{2 p_{1}-1} P_{\zeta} \\
P \equiv P(\zeta, X), \quad P_{\zeta} \equiv P_{\zeta}(\zeta, X) .
\end{gathered}
$$

We obtain a determinant with $4 p_{1}-1$ columns and rows. Every term which lies in the $2 n$th row, and $n$th column, $n$, is of degree 2 , for $n=1,2, \cdots, 2 p_{1}-1$. $P_{\zeta}$ does not contain $\rho^{2}$. Hence the factor $\rho^{2}$ does not appear on any other place except in the above-mentioned terms, and in order to get the highest degree in $\rho$ we have to use the product of these terms. Furthermore, we must take terms of degree one from the next $p_{1}$ columns. Let us now consider the $2 p_{1}$ th column. If we take a term of degree one from this column, then we have taken nothing so far from the first row of the determinant and in all succeeding columns the elements in the first row are 0 . Hence, in order to get a nonvanishing term, we must take in the $2 p_{1}$ th column the term in the first row which is of degree 0 . Hence, we lose 1 degree and the highest possible degree is

$$
2 \cdot\left(2 p_{1}-1\right)+1 \cdot\left(p_{1}-1\right)+0=5 p_{1}-3 .
$$

\section{Harmonic functions of the class $\subseteq$.}

Definition 5.1. The subclass of harmonic functions which can be represented in the form (4.4), (4.5), (4.5a) where $\theta_{1}(\zeta)=\theta_{1}$ and $\phi_{1}(\zeta)=\phi_{1}$ are constants will be denoted by $\subseteq$.

The harmonic functions $H(X) \in \subseteq, X=\left(x_{1}, x_{2}, x_{3}\right)$, are functions of two variables $\rho$ and $T$, see $(4.5 \mathrm{a})$.

If we substitute for $x_{1}, x_{2}, x_{3}$ the values (4.1), the $\mathbf{C}_{3}$-associate of $H \in \subseteq$

(19) They may degenerate in the real $X$-space. 
becomes

$$
\mathbf{C}_{3}^{-1}(H)=\int_{\zeta_{1}}^{\zeta_{2}} \frac{Q(\zeta) d \zeta}{[R(\zeta)(-2 S+R(\zeta))]^{1 / 2}}
$$

where

$$
S=2\left(Z Z^{*}\right)^{1 / 2} \cos \theta_{1}-i \sin \theta_{1} Z e^{i \phi_{1}}-i \sin \theta_{1} Z^{*} e^{-i \phi_{1}} .
$$

Here $\theta_{1}$ and $\phi_{1}$ are constants so that (5.2) becomes a function of one variable, $S$. Since the $\mathbf{C}_{3}$-associate of every function is uniquely determined, the class $\subseteq$ can be characterized by the fact that $\mathrm{C}_{3}{ }^{-1}(\mathfrak{S})$ consists of functions (5.1) of one variable, $S$, of the form (5.2).

The corresponding harmonic functions become functions of two variables $\rho$ and $T$, of the form (4.4), see also (4.5). (Since $\theta_{1}$ and $\phi_{1}$ are constants, $T$ can be considered as a new independent variable.)

In the following we shall: (I) describe the structure of the singularities of the functions of the class S, and (II) show that the coefficients of their series developments at infinity and at the origin lie in certain subspaces of the coefficient space.

Let us assume that the coefficient of the highest power of $\zeta$ in $R(\zeta)$, see (4.5), is 1 . Then for $H \in \mathfrak{S}$, see definition 5.1, let

$$
R(\zeta)=\prod_{j=1}^{p}\left(\zeta-a_{j}\right)
$$

and let

$$
Q(\zeta)=\sum_{\nu=0}^{N} \epsilon_{\nu} \zeta^{\nu}
$$

be a polynomial in $\zeta$ of degree $\leqq N$.

Definition 5.2. The subclass $\subseteq$ of functions (4.4), determined by $R$ and $Q$ of the above form, for which the degree of $Q(\zeta)$ is $\leqq N$ will be denoted by $\mathfrak{A}\left(a_{1}, \cdots, a_{p} ; N\right)$.

Lemma 5.1. The only possible singularity curves of the functions $H(X)$ $\in \mathfrak{A}\left(a_{1}, \cdots, a_{p} ; N\right)$ are $p+1$ circles (which may degenerate) each of which is the intersection of the plane

$$
x_{1} \cos \theta_{1}+x_{2} \sin \theta_{1} \cos \phi_{1}+x_{3} \sin \theta_{1} \sin \phi_{1}=\operatorname{Re}\left(R\left(\zeta^{(k)}\right)\right)
$$

with the sphere

$$
\left(x_{1}-\operatorname{Re}\left(R\left(\zeta^{(k)}\right) \cos \theta_{1}\right)^{2}+\left(x_{2}-\operatorname{Re}\left(R\left(\zeta^{(k)}\right)\right) \sin \theta_{1} \cos \phi_{1}\right)^{2}+\left(x_{3}\right.\right.
$$

$$
-\operatorname{Re}\left(R\left(\zeta^{(k)}\right) \sin \theta_{1} \sin \phi_{1}\right)^{2}=\left(\operatorname{Im} R\left(\zeta^{(k)}\right)\right)^{2} ;
$$

and the straight line consisting of the rays 


$$
\phi=\phi_{1}, \quad \theta=\theta_{1} \quad \text { and } \quad \phi=\phi_{1}+\pi, \quad \theta=-\theta_{1} .
$$

Here $\zeta^{(k)}, k=1,2, \cdots, p-1$, are the $(p-1)$ solutions of $R^{\prime}(\zeta)=0$ and $\zeta^{(p)}=\zeta_{1}, \zeta^{(p+1)}=\zeta_{2}$.

Proof. The equations $P(\zeta, X)=0$, see (4.5), and $P_{\zeta}(\zeta, X)=0$ in this case assume the form

$$
\begin{gathered}
\rho^{2}+R(\zeta)[-2 T \rho+R(\zeta)]=0, \\
T=\cos \theta \cos \theta_{1}+\sin \theta \sin \theta_{1} \cos \left(\phi-\phi_{1}\right), \\
R^{\prime}(\zeta)[-2 T \rho+2 R(\zeta)]=0,
\end{gathered}
$$

respectively. There are two possible types of solutions of (5.7):

(1) $R^{\prime}(\zeta)=0$ : We obtain $p-1$ (generally) distinct values for $\zeta$, say $\zeta=\zeta^{(k)}, \kappa=1,2, \cdots, p-1$. Substituting these values into (5.6) gives $p-1$ circles. Adding two circles corresponding to the end points $\zeta_{1}$ and $\zeta_{2}$, we obtain $p+1$ circles.

(2) $R(\zeta)=\rho T$ : substituting this into (5.6), we obtain $\rho^{2}\left(1-T^{2}\right)=0 . T^{2}$ is always less than or equal to one, and may equal one only if $\phi=\phi_{1}, \theta=\theta_{1}$, and $\phi=\phi_{1}+\pi, \theta=-\theta_{1}$. All other possibilities lead to a different representation of the same line. The origin lies also on this line so that the case $\rho=0$ is already included. This proves our lemma.

Before proceeding further, it will be of interest to discuss in detail the simplest example, namely where $R(\zeta)=A+B \zeta$ is of the first degree. In this case, we can carry out the integration and we obtain

$$
H(X)=\sum_{k=1}^{2}(-1)^{\star} F_{k}
$$

$$
F_{k}=\log \left[C_{k}+\left(C_{k}^{2}+D\right)^{1 / 2}\right], C_{k}=B \zeta_{k}+A+\rho T, D=\rho^{2}\left(1-T^{2}\right) .
$$

We shall determine the singularities of $F_{k}$ in the real (finite) space, $x^{2}+y^{2}+z^{2}<\infty$. The only singular manifolds which can possibly occur correspond to the following four possibilities:

$$
\begin{aligned}
& \text { (5.9.1) } \quad C_{\kappa}=0, D \neq 0, \\
& \text { (5.9.3) } \quad C_{\kappa}=0, D=0,
\end{aligned}
$$$$
\text { (5.9.2) } C_{x} \neq 0, D=0 \text {, }
$$$$
\text { (5.9.4) } C_{k}^{2}+D=0, C_{k} \neq 0 \text {. }
$$

In the first case, the function is regular on the manifold (5.9.1), since the function $F_{x}$ has the development $2^{-1} \log D+C+\cdots$. It can be singular only for $D=0$, i.e., in the case (5.9.3).

The expression in brackets on the right-hand side of (5.8a) is two-valued. In the case (5.9.2), we obtain in the second sheet a logarithmic branch line for $D=0$, i.e., for $\left[\phi=\phi_{1}, \theta=\theta_{1}\right]$ and $\left[\phi=\phi_{1}+\pi, \theta=-\theta_{1}\right]$, where we have a branchline of infinite order. 
The third case, (5.9.3), can occur only if $R\left(\zeta_{k}\right)$ is real, and we obtain an isolated point $\theta=\theta_{1}, \phi=\phi_{1}, \rho=-R\left(\zeta_{k}\right)$. (This point lies on the line $D=0$.) The case (5.9.4) leads to the circle $P\left(\zeta_{k}, X\right)=0$ on which the function is continuous but on which the derivatives are infinite. We obtain, for instance,

$$
\frac{\partial H}{\partial \rho}=\frac{T P\left(\zeta_{\kappa}\right)+2 \rho-\rho T^{2}}{C_{\kappa}\left(C_{\kappa}^{2}+D\right)^{1 / 2}}+\text { reg. terms. }
$$

In the case where $R(\zeta)$ is of a higher degree, the functions can be represented in closed form using hyperelliptic integrals, and a similar discussion can be carried out.

REMARK 5.1. If $H(X) \in \subseteq$, then the location of its singularities and the value of $R(0)$ determine the quantities $a_{1}, \cdots, a_{p}$ in (5.3).

We proceed now to the problem II.

Outside of a sufficiently large sphere, we can develop a function (4.4) of the class $\mathfrak{S}$ in the form of an infinite series of spherical harmonics

$$
\begin{aligned}
& \sum_{n=0}^{\infty} \beta_{n} \rho^{-(n+1)}\left[P_{n}(\cos \theta) P_{n}\left(\cos \theta_{1}\right)\right. \\
& \left.\quad+2 \sum_{\nu=1}^{n} \frac{(n-\nu) !}{(n+\nu) !} P_{n}^{\nu}(\cos \theta) P_{n}^{\nu}\left(\cos \theta_{1}\right)\left(\cos \nu \phi \cos \nu \phi_{1}+\sin \nu \phi \sin \nu \phi_{1}\right)\right] .
\end{aligned}
$$

Definition 5.3. An expression depending only on the $a_{j}, j=1,2, \cdots, p$, and $N$ will be said, for the sake of brevity, to belong to $a_{N}$.

Theorem 5.1. A necessary condition that the series (5.10), where $\theta_{1}$ and $\phi_{1}$ are arbitrary, real constants, represent a functions element (at infinity) of a function of class $\mathfrak{A}\left(a_{1}, \cdots, a_{p} ; N\right)$, see Definition 5.2 , is that for every integer $R \geqq R_{\infty}(p, N) \equiv 6(N+1)(N+2)(N+3) p+7$, there exist a set of numbers $\left\{A_{\rho, \sigma, \tau}^{(R, s)}\right\}, s=1,2, \cdots, s_{R} ; R=R_{\infty}, R_{\infty}+1, \cdots ; A_{\rho, \sigma, \tau}^{(R, s)} \equiv A_{\rho, \sigma, \tau}^{(R, s)}\left(a_{j}, N\right) \in a_{N}$, so that the relations

$$
\sum_{\rho+\sigma+\tau \leqq R} A_{\rho, \sigma, \tau}^{(R, s)} \beta_{\rho} \beta_{\sigma} \beta_{\tau}=0, \quad s=1,2, \cdots, s_{R}, R=R_{\infty}, R_{\infty}+1, \cdots
$$

$\operatorname{hold}\left({ }^{20}\right)$. Here $A_{\rho, \sigma, \tau}^{(R, s)}, s=1,2, \cdots, s_{R}$, for $\rho+\sigma+\tau=R$ are not all zero $\left({ }^{21}\right)$.

Proof. Since $\left(1-2 \rho^{-1} T R(\zeta)+\rho^{-2} R^{2}(\zeta)\right)^{-1 / 2}$, with $T=\cos \alpha$ and $R(\zeta)=1$, is the generating function of the spherical harmonics, we obtain that $\beta_{n}$ in (5.10) are given by

${ }^{(20)} A_{\rho, \sigma, \tau}^{(R, s)}$ are independent of $\epsilon_{\lambda}, \lambda=1,2, \cdots, N$, as well as of $\zeta_{1}$ and $\zeta_{2}$. Explicit formulas for $A_{\rho, \sigma, \tau}^{(R, 0)}, B_{\rho, \sigma, \tau}^{(R, 0)}$ (see Theorem 6.1), as well as for some other quantities considered in the following, can be obtained by straightforward computation. Since they are quite involved, they have been omitted from the present publication. They can be found in the Technical Report No. 32 of the series Studies in Partial Differential Equations, Cambridge, 1951.

(21) This implies that we have infinitely many distinct relations (5.11). 


$$
\beta_{n}=\int_{\zeta_{1}}^{\zeta_{2}} Q(\zeta)[R(\zeta)]^{n} d \zeta
$$

where $Q$ and $R$ are polynomials, see (5.3) and (5.4), so that

$$
\beta_{n}=\sum_{j=0}^{n(p-1)+N} \sum_{\lambda=0}^{N} F(j, \lambda, n) \epsilon_{\lambda}\left[\left(\zeta_{2}-a_{1}\right)^{n+j+1}-\left(\zeta_{1}-a_{1}\right)^{n+j+1}\right],
$$

where $F(j, \lambda, n)$ are certain functions of the $a_{j}$. (See footnote 20.) Our aim is to obtain relations among the $\beta_{n}$ 's which depend only on $a_{j}$, but are independent of $\zeta_{1}, \zeta_{2}$, as well as the coefficients $\epsilon_{\lambda}$ of $Q$. For this purpose, we multiply three $\beta_{n}$ 's by each other, and get relations of the form

$$
\begin{aligned}
\beta_{\rho} \beta_{\sigma} \beta_{\tau}= & \sum_{\lambda_{1}=0}^{N} \sum_{\lambda_{2}=0}^{\lambda_{1}} \sum_{\lambda_{3}=0}^{\lambda_{2}} \sum_{\alpha=1}^{[(R p+3(N+1)) / 2]} \sum_{\beta=0}^{\alpha} S\left(\alpha, \beta ; \lambda_{1}, \lambda_{2}, \lambda_{3} ; \rho, \sigma, \tau\right) \\
& \times\left\{\left(\zeta_{2}-a_{1}\right)^{\alpha}\left(\zeta_{1}-a_{1}\right)^{\beta}-\left(\zeta_{1}-a_{1}\right)^{\alpha}\left(\zeta_{2}-a_{1}\right)^{\beta}\right\} \epsilon_{\lambda_{1}} \epsilon_{\lambda_{2}} \epsilon_{\lambda_{8}},
\end{aligned}
$$

where $S \in a_{N}$. In the following, we shall consider the expressions

$$
\left\{\left(\zeta_{2}-a_{1}\right)^{\alpha}\left(\zeta_{1}-a_{1}\right)^{\beta}-\left(\zeta_{1}-a_{1}\right)^{\alpha}\left(\zeta_{2}-a_{1}\right)^{\beta}\right\} \epsilon_{\lambda_{1}} \epsilon_{\lambda_{2}} \epsilon_{\lambda_{3}}
$$

as variables which eventually have to be eliminated from the equations (5.14). To prove Theorem 5.1, we need a number of lemmas which are for the most part evident.

LEMмA 5.2. The number of non-negative integers satisfying the inequalities

$$
0 \leqq \lambda_{1} \leqq \lambda_{2} \leqq \lambda_{3} \leqq N
$$

is $(N+1)(N+2)(N+3) / 6$.

LEMмA 5.3. The number of distinct ways of writing an integer $R>2$ as $a$ sum of three positive integers is larger than $R^{2} / 6$.

LemмA 5.4. If $R>(N+1)(N+2)(N+3) p$, then the number of variables (5.14a) for which $\alpha+\beta$ appearing as exponents in the right-hand side of (5.14) assumes its maximum value $R p+3(N+1)$ is less than the number of equations (5.14) for which $\rho+\sigma+\tau=R$.

Proof. By Lemma 5.3, the number of equations is larger than $R^{2} / 6$. The number of variables is

$$
\frac{(N+1)(N+2)(N+3)}{6}\left\{1+\frac{R p+3(N+1)}{2}\right\} .
$$

If $R$ satisfies the conditions of Lemma 5.4 , it is obvious that $R^{2} / 6$ is larger than (5.15).

We now estimate the number of triples $(\rho, \sigma, \tau)$ such that $\rho+\sigma+\tau \leqq R$ 
and $0 \leqq \rho \leqq \sigma \leqq \tau$.

The number of distinct ways of writing an integer $M$ as the sum of two positive integers is $\geqq[M / 2]\left({ }^{22}\right)$. Hence the number of distinct ways of writing all positive integers $\leqq M$ as a sum of two positive integers is larger or equal to

$$
\left[\frac{M}{2}\right]+\left[\frac{M-1}{2}\right]+\left[\frac{M-2}{2}\right]+\cdots+\left[\frac{1}{2}\right] \geqq\left[\frac{M}{2}\right]^{2} \text {. }
$$

We now proceed to the determination of the number of distinct ways of writing all integers $\leqq M$ as the sum of three positive integers. Suppose that the smallest of these three integers is $K$. Then the number of triples whose smallest member is $K$ is the same as the number of distinct pairs adding up to $M-K$ where each member of these pairs must be larger or equal to $K$. This number is by (5.16) larger than

$$
[(M-3 K) / 2]^{2} \text {. }
$$

If $K$ varies from 1 to $[M / 3]$ (since $K$ is the smallest of the triple, $K \leqq[M / 3]$ ) we obtain all possible triples. It remains to determine a lower bound for

$$
\sum_{K=0}^{(M / 3)}[(M-3 K) / 2]^{2}
$$

We shall show that for $M \geqq 36$

$$
(M-7)^{3} / 36
$$

is such a lower bound. If we write the sequence $[(M-3 K) / 2]^{2}, K$ $=0,1,2, \cdots$, then we obtain a consecutive series of squares decreasing from $[M / 2]^{2}$ to $1^{2}$, with every third term missing. The worst possibility is if the numbers $[(M-2-6 \kappa) / 2]^{2}, \kappa=0,1, \cdots,[(M-9) / 6]^{2}$, are missing. We obtain therefore that (5.18) is greater than or equal to

$$
\begin{aligned}
\sum_{n=1}^{[M / 2]} n^{2}-9 & \sum_{n=1}^{[(M-2) / 6]} n^{2}=\frac{1}{6}\{[M / 2]([M / 2]+1)(2[M / 2]+1) \\
& \quad-9[(M-2) / 6]([(M-2) / 6]+1)(2[(M-2) / 6]+1)\} \\
\geqq & \frac{1}{6}\left\{\frac{1}{4}(M-3)^{3}-\frac{1}{12}(M+3)^{3}\right\} \geqq \frac{1}{36}(M-7)^{3}, \text { for } M \geqq 36 .
\end{aligned}
$$

Thus, if $\rho, \sigma, \tau$ covers all distinct possibilities so that $\rho+\sigma+\tau \leqq R$, then the number of equations is larger than $(R-7)^{3} / 36$. On the other hand, adding expressions (5.15) for $R=1, \cdots, R=R\left({ }^{23}\right)$, we see that the number of dis-

(22) $[S]$ denotes the largest integer which is smaller or equal to $S$.

${ }^{(23}$ In order to avoid subscripts we use the same symbol $R$ both as a summation index and as the upper limit. 
tinct variables $(5.14 \mathrm{a})$ in these equations is

$$
\frac{(N+1)(N+2)(N+3)}{24} R(R p+10+6 N+p) \text {. }
$$

A simple calculation shows that under the hypothesis of Theorem 5.1,

$$
\frac{1}{36}(R-7)^{3}>\frac{(N+1)(N+2)(N+3)}{24} R(R p+10+6 N+p) \text {. }
$$

Indeed: $R<2(R-7)$ if $R>14$, and $R p+10+6 N+p<2(R p-7 p)$ if $R>25$ $+6 N$. In addition to that, according to (5.20), $R \geqq 36$. It is now clear that if $R>6(N+1)(N+2)(N+3) p+7$, then (5.22) is satisfied, and this condition implies the three previous ones.

We wish to show that if we have $m$ equations

$$
\sum_{\mu=1}^{n} A_{\nu \mu} X_{\mu}=L_{\nu}, \quad \nu=1, \cdots, m,
$$

in which $n$ variables $X_{\mu}$ occur, $m>n$, then, necessarily, we obtain at least $(m-n)$ distinct relations among the $A_{\nu \mu}$ and $L_{\nu}$. They are linear in $L_{\nu}$ and lead to (5.11). If we write $X_{n+1}=1$, the system (5.23) becomes

$$
\sum_{\mu=1}^{n} A_{\nu \mu} X_{\mu}-L_{\nu} X_{n+1}=0, \quad \nu=1,2, \cdots, m .
$$

Since $m \geqq n+1$ and since we know that not all $X_{\mu}, \mu=1, \cdots, n+1$, are identically equal to zero, the determinant of any system of $(n+1)$ equations (5.24) equals zero. This yields at least one relation between the $L_{v}$ and the $A_{\nu \mu}$. Thus, we get at least $m-n$ independent relations.

The system of relations (5.14) is of the type (5.23). Here, $L_{\nu}$ are the $\beta_{\rho} \beta_{\sigma} \beta_{\tau}$ 's,

$$
X_{\mu}=\left[\left(\zeta_{2}-a_{1}\right)^{\alpha}\left(\zeta_{1}-a_{1}\right)^{\beta}-\left(\zeta_{1}-a_{1}\right)^{\alpha}\left(\zeta_{2}-a_{1}\right)^{\beta}\right] \epsilon_{\lambda_{1}} \epsilon_{\lambda_{2}} \epsilon_{\lambda_{3}},
$$

and the $A_{\nu \mu}$ are the coefficients $S\left(\alpha, \beta ; \lambda_{1}, \lambda_{2}, \lambda_{3} ; \rho, \sigma, \tau\right)$. The latter belong to the class $a_{N}$, introduced on p. 20. If $R$ is increased in the equations (5.14), new $A_{\nu \mu}$ and $L_{\nu}$ appear. By Lemma 5.4, these new $A_{\nu \mu}$ 's and $L_{\nu}$ 's will actually occur in the determinant of (5.24) which we obtain for every new $R$. Thus at each step we obtain a new relation involving some $A_{\nu \mu}$ 's or $L_{\nu}$ 's, which did not appear previously. This proves Theorem 5.1 .

REMARK 5.2. The $A_{\rho, \sigma, \tau}^{(R, s)}$ for a fixed $R$ are, according to our proof, certain subdeterminants of the matrix of coefficients

$$
\left[S\left(\alpha, \beta ; \lambda_{1}, \lambda_{2}, \lambda_{3} ; \rho, \sigma, \tau\right)\right], \quad \rho+\sigma+\tau \leqq R .
$$

A detailed description of these expressions is given in the appendix.

ExAmple. $p=1, N=0, R(\zeta)=(\zeta-a)$. 
In this case, the harmonic function under consideration is

$$
H(X)=\int_{\zeta_{1}}^{\zeta_{2}} \frac{d \zeta}{\left[\rho^{2}+(\zeta-a)^{2}-2 \rho(\zeta-a)\left(\cos \theta \cos \theta_{1}+\sin \theta \sin \theta_{1} \cos \left(\phi_{1}-\phi\right)\right)\right]^{1 / 2}} .
$$

Then the coefficients $\beta_{n}$ of the development (5.10) at infinity satisfy an infinite number of relations (5.11), of which the first two are:

$$
\begin{aligned}
& \beta_{0}^{2} \beta_{3}-3 \beta_{0} \beta_{1} \beta_{2}+2 \beta_{1}^{3}=0, \\
& 5 \beta_{0}^{2} \beta_{4}-8 \beta_{0} \beta_{1} \beta_{3}-9 \beta_{0} \beta_{2}^{2}+12 \beta_{1}^{2} \beta_{2}=0 .
\end{aligned}
$$

6. The development of a function of the class $\subseteq$ at the origin, and its properties. In a sufficiently small neighborhood of the origin, we can develop the function (4.4) in a series of spherical harmonics

$$
\begin{aligned}
H(X)= & \int_{\zeta_{1}}^{\zeta_{2}} \frac{Q(\zeta) d \zeta}{(P(\zeta, X))^{1 / 2}} \\
= & \int_{\zeta_{1}}^{\zeta_{2}} \sum_{n=0}^{\infty} \frac{Q(\zeta) d \zeta}{(R(\zeta))^{n+1}} \rho^{n} P_{n}\left(\cos \theta \cos \theta_{1}+\sin \theta \sin \theta_{1} \cos \left(\phi_{1}-\phi\right)\right) \\
= & \int_{\zeta_{1}}^{\zeta_{2}} \sum_{n=0}^{\infty} \frac{Q(\zeta) d \zeta}{(R(\zeta))^{n+1}} \rho^{n}\left[P_{n}(\cos \theta) P_{n}\left(\cos \theta_{1}\right)\right. \\
& +2 \sum_{\nu=1}^{n} \frac{(n-\nu) !}{(n+\nu) !} P_{n}^{\nu}(\cos \theta) P_{n}^{\nu}\left(\cos \theta_{1}\right)\left(\cos \nu \phi \cos \nu \phi_{1}\right.
\end{aligned}
$$

$\left.\left.+\sin \nu \phi \sin \nu \phi_{1}\right)\right]$.

(See $[13$, vol. 1, p. 400, (44)].)

Thus the coefficient of the term $\rho^{n} P_{n}(\cos \theta)$ is

$$
\gamma_{n+1} P_{n}\left(\cos \theta_{1}\right), \quad \gamma_{n+1}=\int_{\zeta_{1}}^{\zeta_{2}} \frac{Q(\zeta) d \zeta}{(R(\zeta))^{n+1}},
$$

while the coefficient of $\rho^{n} P_{n}^{\nu}(\cos \theta) \cos \nu \phi$ is

$$
2 \gamma_{n+1} \frac{(n-\nu) !}{(n+\nu) !} P_{n}^{\nu}\left(\cos \theta_{1}\right) \cos \nu \phi_{1} .
$$

Theorem 6.1. A necessary condition that the series

$$
\sum_{n=0}^{\infty} \rho^{n}\left\{\tau_{n} P_{n}(\cos \theta)+\sum_{\nu=1}^{n} P_{n}^{\nu}(\cos \theta)\left[\tau_{n \nu}^{(1)} \cos \nu \phi+\tau_{n \nu}^{(2)} \sin \nu \phi\right]\right\}
$$

represent a series development at the origin of a function of the class $\mathfrak{A}\left(a_{1}, \cdots, a_{p} ; N\right)$ is that there exist constants $\theta_{1}$ and $\phi_{1},\left|\theta_{1}\right| \leqq \pi / 2,\left|\phi_{1}\right| \leqq \pi$, and $a$ series of numbers $\gamma_{n}, n=1,2, \cdots$, such that 


$$
\begin{aligned}
\tau_{n} & =\gamma_{n+1} P_{n}\left(\cos \theta_{1}\right), \\
(n+\nu) ! \tau_{n \nu}^{(1)} & =2 \gamma_{n+1}(n-\nu) ! P_{n}^{\nu}\left(\cos \theta_{1}\right) \cos \nu \phi_{1}, \\
(n+\nu) ! \tau_{n \nu}^{(2)} & =2 \gamma_{n+1}(n-\nu) ! P_{n}^{\nu}\left(\cos \theta_{1}\right) \sin \nu \phi_{1},
\end{aligned}
$$

and that for every integer $R \geqq R_{0}(p, N) \equiv 3(N+1) p\left[2(N+2)(N+3) p^{2}+1\right]+7$, there exists a series of numbers $B_{\rho, \sigma, \tau}^{(R, s)} \in a_{N}$ (see Definition 5.3 and footnote 20) so that

$$
\sum_{\rho+\sigma+\tau \leqq R} B_{\rho, \sigma, \tau}^{(R, 8)} \gamma_{\rho} \gamma_{\sigma} \gamma_{\tau}=0, \quad s=1,2, \cdots, s_{R} ; R=R_{0}, R_{0}+1, \cdots
$$

Here $B_{\rho, \sigma, \tau}^{(R, s)}, s=1,2, \cdots, s_{R}$, for $\rho+\sigma+\tau=R$ are not all zero $\left({ }^{24}\right)$.

Proof. The proof is in principle similar to that of Theorem 5.1, but some modifications have to be discussed. In this case,

$$
\gamma_{n}=\int_{\zeta_{1}}^{\zeta_{2}} \frac{Q(\zeta)}{(R(\zeta))^{n}} d \zeta=\int_{\zeta_{1}}^{\zeta_{2}} \frac{Q(\zeta) d \zeta}{\prod_{\kappa=1}^{p}\left(\zeta-a_{\kappa}\right)^{n}}
$$

The variables $Y_{n}$ which we shall eliminate are in this case

$$
\begin{gathered}
Y_{n}=\left[\left(\zeta_{1}-a_{i}\right)^{-\alpha}\left(\zeta_{2}-a_{j}\right)^{-\beta}-\left(\zeta_{2}-a_{i}\right)^{-\alpha}\left(\zeta_{1}-a_{j}\right)^{-\beta}\right] \epsilon_{\lambda_{1}} \epsilon_{\lambda_{2}} \epsilon_{\lambda_{3}}, \\
\alpha \leqq \beta, \quad 0 \leqq \lambda_{1} \leqq \lambda_{2} \leqq \lambda_{3} \leqq N .
\end{gathered}
$$

The following three additional complications arise in this case.

(1) Since, say, $\left(\zeta_{1}-a_{j}\right)^{\alpha}$ now appear in the denominator we can not express it in finitely many terms $\left(\zeta_{1}-a_{1}\right)^{-\alpha_{\nu}}$ of the same form. Hence, a much larger number of variables $Y_{n}$ have to be eliminated.

(2) In order to express the integrand of $\gamma_{n}$ in terms of the variables $Y_{n}$ we have to use partial fractions. Furthermore, after we multiply three $\gamma_{n}$ together, we again have used partial fractions to express $\gamma_{\rho} \gamma_{\sigma} \gamma_{\tau}$ in terms of $Y_{n}$. This situation makes the coefficients of the $Y_{n}$ very much more complicated.

(3) After we express the integrand of $\gamma_{n}$ in partial fractions and integrate, we obtain $p(N+1)$ distinct logarithmic terms. Using the first $p(N+1)$ formulas for $\gamma_{n}$ we can represent these logarithmic expressions in terms of $\gamma_{n}, n=1,2, \cdots, p(N+1)$. The logarithmic terms in the formulas for $\gamma_{\rho}, \rho>p(N+1)$, must be replaced by these terms, before we form the expressions $\gamma_{\rho} \gamma_{\sigma} \gamma_{\tau}$.

As a consequence of these three complicating factors, the coefficients $B_{\rho, \sigma, \tau}^{(R, s)}$ in the relations (6.4) will be much more involved and $R_{0}(N, p)$ will be very much larger than $R_{\infty}(N, p)$.

In order to evaluate (6.5), we separate the integrand in partial fractions,

(24) This implies that we have infinitely many distinct relations (6.4). 
and we obtain $\left({ }^{25}\right)$

$$
\begin{aligned}
\gamma_{n}= & \sum_{\nu=0}^{N} \sum_{k=1}^{p} \sum_{j=1}^{n} \int_{\zeta_{1}}^{\zeta_{2}} \frac{A_{\kappa, j, \nu}^{(n)} \epsilon_{\nu}}{\left(\zeta-a_{k}\right)^{j}} d \zeta \\
= & -\sum_{\nu=0}^{N} \sum_{k=1}^{p} \sum_{j=2}^{n} \frac{A_{k, j, \nu}^{(n)} \epsilon_{\nu}}{j-1}\left[\left(\zeta_{2}-a_{k}\right)^{-j+1}-\left(\zeta_{1}-a_{k}\right)^{-j+1}\right] \\
& +\sum_{\nu=0}^{N} \sum_{\kappa=1}^{p} A_{\kappa, 1, \nu}^{(n)} \epsilon_{\nu} \log \frac{\left(\zeta_{2}-a_{k}\right)}{\left(\zeta_{1}-a_{k}\right)} .
\end{aligned}
$$

$\operatorname{Here} A_{\kappa, j, \nu}^{(n)} \in a_{N}$

The expressions $\gamma_{n}$ all contain the same $(N+1) p$ logarithmic terms.

REMARK. Here we assume that the determinant

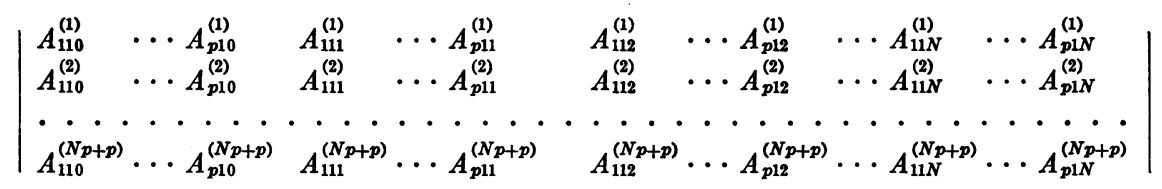

does not vanish. Should this not be the case, then we have to use more expressions(6.7).

If we use the $(N+1) p$ first relations (6.7) the $\epsilon_{\nu} \log \left[\left(\zeta_{2}-a_{\kappa}\right) /\left(\zeta_{1}-a_{\kappa}\right)\right]$ can be expressed in terms of $\gamma_{n}, n \leqq(N+1) p$, and rational terms, i.e., we have

$$
\begin{aligned}
\epsilon_{\nu} \log \frac{\left(\zeta_{2}-a_{k}\right)}{\left(\zeta_{1}-a_{k}\right)} & =\sum_{m=1}^{(N+1) p} \tau_{m} \gamma_{m} \\
& +\sum_{n=2}^{(N+1) p} \sum_{\nu=0}^{N} \sum_{k=1}^{p} \sum_{j=2}^{n} B_{k, j, \nu}^{(n)}\left[\left(\zeta_{2}-a_{k}\right)^{-j+1}-\left(\zeta_{1}-a_{k}\right)^{-j+1}\right] \epsilon_{\nu}
\end{aligned}
$$

where $\tau_{m}$ and $B_{\kappa, j, \nu}^{(n)}$ belong to $a_{N}$. (See Definition 5.3.)

Replacing for every $n>(N+1) p$ the logarithmic terms in (6.7) by (6.8), we obtain that a certain linear combination

$$
\delta_{n}=\gamma_{n}-\sum_{m=1}^{(N+1) p} \Delta_{m}^{(n)} \gamma_{m} ; \quad \Delta_{m}^{(n)} \in a_{N}
$$

is a rational expression,

$$
\begin{aligned}
\delta_{n}=\sum_{k=1}^{p} \sum_{j=2}^{n} \sum_{\nu=0}^{N}\left[\begin{array}{rr}
\nu, & n \\
\kappa, & j
\end{array}\right] & {\left[\left(\zeta_{2}-a_{k}\right)^{1-j}-\left(\zeta_{1}-a_{k}\right)^{1-j}\right] \epsilon_{\nu}, } \\
& n=(N+1) p+1,(N+1) p+2, \cdots ;
\end{aligned}
$$

(25) Explicit formulas for the $A_{\kappa, j, \nu}^{(n)}$ as well as for all subsequent expressions may be found in the article mentioned in footnote 20 . 


$$
\left[\begin{array}{ll}
\nu, & n \\
\kappa, & j
\end{array}\right]
$$

being some functions belonging to $a_{N}$.

By multiplying two $\delta_{n}$ we obtain, as we shall prove,

$$
\begin{aligned}
\delta_{\rho} \delta_{\sigma}= & \sum_{\mu_{1}=0}^{N} \sum_{\mu_{2}=0}^{\mu_{1}} \epsilon_{\mu_{1}} \epsilon_{\mu_{2}}\left\{\sum_{\kappa=1}^{p} \sum_{\nu=1}^{\rho+\sigma-2} G\left(\kappa, \nu, \mu_{1}, \mu_{2} ; \rho, \sigma\right)\right. \\
& \times\left[\left(\zeta_{2}-a_{\kappa}\right)^{-\nu}+\left(\zeta_{1}-a_{\kappa}\right)^{-\nu}\right] \\
& -\sum_{i_{1}=1}^{p} \sum_{i_{2}=1}^{p} \sum_{j_{1}=2}^{\rho} \sum_{j_{2}=2}^{\sigma}\left[\begin{array}{l}
\mu_{1}, \rho \\
i_{1}, j_{1}
\end{array}\right]\left[\begin{array}{l}
\mu_{2}, \sigma \\
i_{2}, j_{2}
\end{array}\right]\left(\left(\zeta_{1}-a_{i_{1}}\right)^{-j_{1}+1}\left(\zeta_{2}-a_{i_{2}}\right)^{-j_{2}+1}\right. \\
& \left.\left.+\left(\zeta_{1}-a_{i_{2}}\right)^{-j_{2}+1}\left(\zeta_{2}-a_{i_{1}}\right)^{-j_{1}+1}\right)\right\}
\end{aligned}
$$

where $G\left(\kappa, \nu, \mu_{1}, \mu_{2} ; \rho, \sigma\right) \in a_{N}$.

Proof of (6.11). By multiplying two expressions of the form (6.10) for $n=\rho$ and $n=\sigma$, we obtain on the right-hand side sums of the following types of expressions:

1. $\left(\zeta_{1}-a_{i_{1}}\right)^{-j_{1}}\left(\zeta_{1}-a_{i_{2}}\right)^{-i_{2}}, i_{1} \neq i_{2}$, and analogous expressions in $\zeta_{2}$ (instead of $\zeta_{1}$ ). Applying the method of partial fractions to these terms, we obtain expressions appearing in the first sum in the bracket in (6.11).

2. If $i_{1}=i_{2}$ the above expression becomes $\left(\zeta_{1}-a_{i_{1}}\right)^{-\left(i_{1}+i_{2}\right)}$ from which we obtain again expressions of the same form.

3. Mixed terms of the form $\left(\zeta_{1}-a_{i_{1}}\right)^{-j_{1}}\left(\zeta_{2}-a_{i_{2}}\right)^{-j_{2}}$ which cannot be separated in any way are collected in the last sum of (6.11).

Multiplying three $\delta_{n}$ together, we obtain

$$
\delta_{\rho} \delta_{\sigma} \delta_{\tau}=\sum_{\mu_{1}=0}^{N} \sum_{\mu_{2}=0}^{\mu_{1}} \sum_{\mu_{3}=0}^{\mu_{2}} \epsilon_{\mu_{1}} \epsilon_{\mu_{2}} \epsilon_{\mu_{3}}\left(S_{1}\left(\mu_{1}, \mu_{2}, \mu_{3}\right)+S_{2}\left(\mu_{1}, \mu_{2}, \mu_{3}\right)\right)
$$

where

$$
\begin{aligned}
& S_{1}\left(\mu_{1}, \mu_{2}, \mu_{3}\right)=\sum_{s=1}^{p} \sum_{\mu=1}^{\rho+\sigma+\tau-3} H\left(s, \lambda ; \mu_{1}, \mu_{2}, \mu_{3} ; \rho, \sigma, \tau\right)\left[\left(\zeta_{2}-a_{s}\right)^{-\mu}-\left(\zeta_{1}-a_{s}\right)^{-\mu}\right], \\
& S_{2}\left(\mu_{1}, \mu_{2}, \mu_{3}\right)=\sum_{s=1}^{p} \sum_{t=1}^{p} \sum_{\mu=1}^{\sigma+\tau-2} \sum_{\lambda=1}^{\min (\tau-1, \rho+\sigma+\tau-3-\mu)} T\left(\mu, s, \lambda, t ; \mu_{1}, \mu_{2}, \mu_{3} ; \rho, \sigma, \tau\right) \\
& \times\left[\left(\zeta_{1}-a_{8}\right)^{-\mu}\left(\zeta_{2}-a_{t}\right)^{-\lambda}-\left(\zeta_{2}-a_{s}\right)^{-\mu}\left(\zeta_{1}-a_{t}\right)^{-\lambda}\right],
\end{aligned}
$$

and the $H$ 's and the $T$ 's $\in a_{N} .(6.12)$ is obtained by repeating the previous arguments.

The proof of the relations (6.4) now proceeds analogously to that of (5.11).

If we compare the variables $X_{\mu}$ (introduced in (5.25)) with the $Y_{\mu}$ (intro- 
duced in (6.6)) we see that they are exactly of the same form, with the only difference that while in (6.6) expressions of the type

$$
\begin{aligned}
{\left[\left(\zeta_{2}-a_{\mathfrak{i}}\right)^{-\alpha}\left(\zeta_{1}-a_{j}\right)^{-\beta}-\left(\zeta_{1}-a_{\mathbf{i}}\right)^{-\alpha}\left(\zeta_{2}-a_{j}\right)^{-\beta}\right] \epsilon_{\mu_{1}} \epsilon_{\mu_{2}} \epsilon_{\mu_{3}}, } \\
\mathrm{i}=1,2, \cdots, p ; j=1,2, \cdots, p,
\end{aligned}
$$

appear, in (5.25) only expressions with $i=1, j=1$ do appear. Therefore, in the case of the $Y_{n}$ we have $p^{2}$ as many variables as in the case of the $X_{n}$. Thus according to (5.21) the number of the variables is

$$
((N+1)(N+2)(N+3) / 24) R(R p+10+6 N+p) p^{2} .
$$

In order to determine the number of equations, we recall the considerations on p. 23 of $\$ 5$. We have, however, to take into account that $\rho, \sigma, \tau$, in addition to the restriction $\rho+\sigma+\tau \leqq R$, must each be larger than $(N+1) p$, since the first $(N+1) p$ relations are used to eliminate the logarithmic terms. Hence, the number of equations will be larger than

$$
[R-3(N+1) p-7]^{3} / 36 \text {. }
$$

In an analogy to the considerations on p. 23 , we find that (6.14) is larger than (6.13) if

$$
R \geqq R_{0}(P, N) \equiv 3(N+1) p\left[2(N+2)(N+3) p^{2}+1\right]+7 .
$$

Repeating analogous considerations as in $\S 5$, we obtain that the $\delta_{\rho} \delta_{\sigma} \delta_{\tau}$ 's satisfy relations of the form (6.4). But since, according to (6.9), the $\delta_{n}$ 's are linear combinations of the $\gamma_{n}$ 's, the relation (6.4) follows.

7. Solutions $\psi(X)$ of $(1.3)$ of the class $\subseteq$ and relations among their coefficients. In (5.26) of [6] (see also (4.3) of [8]) we have introduced the operators $\mathbf{P}_{3}$ and $\mathbf{p}_{3}$ transforming harmonic functions into solutions of $(1.3)\left({ }^{26}\right)$

$$
\begin{aligned}
\psi(X) & =\mathbf{P}_{3}(H) \equiv \int_{-1}^{1} \Omega(\rho, \tau) H\left(X\left(1-\tau^{2}\right)\right) d \tau \\
& =\mathbf{p}_{3}(G) \equiv G(X)+\sum_{n=1}^{\infty} B^{(n)}\left(\rho^{2}\right) \int_{0}^{1}\left(1-\sigma^{2}\right)^{n-1} \sigma^{2} G\left(\sigma^{2} X\right) d \sigma
\end{aligned}
$$

where $\Omega(\rho, \tau)$ and $B^{(n)}\left(\rho^{2}\right)$ are entire functions of $\rho^{2}$. See (4.3) and (4.4) of [8].

Here $G(\boldsymbol{X})$ and $H(\boldsymbol{X})$ are connected by the relations

$$
G(X)=\int_{0}^{1} T^{-1 / 2} H(X(1-T)) d T,
$$

(26) In [6] we used the letter $H$ instead of $\Omega$, employing $\Omega$ in the more general case of equation $\boldsymbol{T}(\psi)=\nabla^{2} \psi+A\left(\rho^{2}\right) \cdot \boldsymbol{X} \cdot \nabla \psi+C\left(\rho^{2}\right) \psi=0$. If $A=0, C=F$, see (2.2) of [6], we obtain the case considered in the present paper. Our results can easily be extended to the case of an equation $T(\psi)=0$ where $A$ and $C$ are entire functions of $\rho^{2}=\sum_{k=1}^{3} x_{k}^{2}$. 
see $(4.8)$ of $[8]$, and

$$
\begin{aligned}
& H(X) \equiv \widetilde{H}(\rho, \theta, \phi)=2 \pi^{-1} \rho^{1 / 2} d\left[\rho^{1 / 2} \int_{0}^{1} \tilde{G}\left(\rho t^{2}, \theta, \phi\right)\left(1-t^{2}\right)^{-1 / 2} d t\right] / d \rho \\
& G(X) \equiv \widetilde{G}(\rho, \theta, \phi) .
\end{aligned}
$$

Proof of (7.2b). Let $G$ and $H$ be the $\mathbf{p}_{3}$-associate and the $\mathbf{P}_{3}$-associate, respectively, of the same solution $\psi$. If we write

$$
G(X) \equiv \widetilde{G}(\rho, \theta, \phi)=\sum_{p=0}^{\infty} \rho^{p} Q_{p}(\theta, \phi)
$$

and $H(\boldsymbol{X})=\widetilde{H}(\rho, \theta, \phi)=\sum_{p=0}^{\infty} \rho^{p} q_{p}(\theta, \phi)$, where $Q_{p}(\theta, \phi)$ and $q_{p}(\theta, \phi)$ are functions of $\theta$ and $\phi,|\theta| \leqq \pi / 2,|\phi| \leqq \pi$, then according to (4.8) of [8]

$$
\begin{aligned}
\sum_{p=0}^{\infty} \rho^{p} q_{p}(\theta, \phi) & =\sum_{p=0}^{\infty} \frac{\Gamma(p+3 / 2)}{\Gamma(p+1) \Gamma(1 / 2)} \rho^{p} Q_{p}(\theta, \phi) \\
& =\frac{\pi}{2} \sum_{p=0}^{\infty}\left(p+\frac{1}{2}\right) \rho^{p} Q_{p}(\theta, \phi) \int_{0}^{1} t^{2 p}\left(1-t^{2}\right)^{-1 / 2} d t,
\end{aligned}
$$

from which (7.2b) follows.

As we have shown in Lemma 4.2, p. 502, of [8]

$$
\psi\left(i\left(x_{2}^{2}+x_{3}^{2}\right)^{1 / 2}, x_{2}, x_{3}\right)=G\left(i\left(x_{2}^{2}+x_{3}^{2}\right)^{1 / 2}, x_{2}, x_{3}\right),
$$

i.e.,

$$
\mathbf{C}_{3}^{-1}(\psi)=\mathbf{C}_{3}^{-1}\left(\mathbf{p}_{3}^{-1}(\psi)\right),
$$

see $\left(4.15\left({ }^{27}\right)\right.$ of $[6]$.

It follows from (7.1) that $\psi(\boldsymbol{X})$ is regular at every point $\boldsymbol{X}$ where $H$ is regular.

Definition 7.1. Extending the definition 5.1 we shall say that solutions of (1.3) whose $\mathbf{C}_{3}$-associates are of the form (5.1) belong to the class $\mathfrak{S}$.

Definition 7.2. If $G(X)=\int_{0}^{1} T^{-1 / 2} H(X(1-T)) d T \in \mathfrak{A}\left(a_{1}, \cdots, a_{p} ; N\right)$, then (extending the definition 5.2) $\psi=\mathbf{P}_{3}(H) \equiv \mathbf{p}_{3}(G)$ will be said to belong to the subclass $\mathfrak{A}\left(a_{1}, \cdots, a_{p} ; N\right) ; \psi \in \mathfrak{A}\left(a_{1}, \cdots, a_{p} ; N\right)$.

From our previous statements follows the

Lemma 7.1. The only (possible) singularities in the finite $X$-space of a solution $\psi(X) \in \mathfrak{H}\left(a_{1}, \cdots, a_{p} ; N\right)$ of $(1.3)$ are the circles $(5.5 \mathrm{a}),(5.5 \mathrm{~b})$, and the straight line (5.5c).

REMARK 7.1. Thus as in the two-dimensional case the location of the

(27) In (4.15) of [8] $b_{3}{ }^{-1}$ should be replaced by $\mathrm{p}_{3}{ }^{-1}$. It should be stressed that, on the lefthand side of $(7.2 \mathrm{~b}), \mathrm{C}_{3}$ refers to the operator transforming $\chi\left(Z, Z^{*}\right)$ into solutions of (1.3), while on the right-hand side it refers to the operator transforming $\chi\left(Z, Z^{*}\right)$ into harmonic functions. 
singularities is completely independent of the coefficient $F$ of (1.3).

LEMMA 7.2. Every real solution of (1.3) which is regular at the origin $O$ can be developed in a sufficiently small neighborhood of $O$ in a series

$$
\begin{aligned}
\psi(X) \equiv \tilde{\psi}(\rho, \theta, \phi)= & \sum_{n=0}^{\infty} \sum_{\nu=0}^{\infty} \rho^{n+\nu} \sum_{m=-n}^{n} B_{m}^{(n, v)} P_{n}^{|m|}(\cos \theta) e^{i m \phi}, \\
& B_{m}^{(n, \nu)}=\bar{B}_{-m}^{(n, \nu)} .
\end{aligned}
$$

The representation (7.4) is unique $\left({ }^{28}\right)$.

Proof. Let

$$
\begin{gathered}
H(X) \equiv \tilde{H}(\rho, \theta, \phi)=\sum_{n=0}^{\infty} \rho^{n}\left[B_{0}^{(n)} P_{n}(\cos \theta)+\sum_{m=-n}^{n} B_{m}^{(n)} P_{n}^{|m|}(\cos \theta) e^{i m \phi}\right], \\
B_{m}^{(n)}=\bar{B}_{-m}^{(n)} .
\end{gathered}
$$

Since according to (3.10), p. 425 of [6],

$$
\Omega(\rho, \tau)=1+\sum_{v=1}^{\infty} \tau^{2 v} b^{(v)}\left(\rho^{2}\right)
$$

it follows from (7.1) that

$$
\begin{aligned}
\psi(X) & =\widetilde{\psi}(\rho, \theta, \phi)=\int_{-1}^{1}\left[1+\rho^{2} \sum_{\nu=1}^{\infty} \tau^{2 \nu} b^{(\nu)}\left(\rho^{2}\right) \rho^{-2}\right] \\
& \times\left[\sum_{n=0}^{\infty} \rho^{n}\left(1-\tau^{2}\right)^{n}\left(A_{n 0} P_{n}(\cos \theta)+\sum_{m=-n}^{n} B_{m}^{(n)} P_{n}^{|m|}(\cos \theta) e^{i m \phi}\right)\right] d \tau
\end{aligned}
$$

where $\rho^{-2} b^{(n)}\left(\rho^{2}\right)$ are according to (3.9), p. 425, of [6] entire functions of $\rho^{2}$.

Both series (7.5) and (7.6) converge uniformly and absolutely in every sufficiently small sphere around the origin (see Lemma 3.2 of [6]); hence, interchanging of the order of summation and integration is permissible. Thus

$$
\begin{aligned}
\psi(X)=\sum_{n=0}^{\infty} \rho^{n}\left(\int _ { - 1 } ^ { 1 } ( 1 - \tau ^ { 2 } ) ^ { n } d \tau \left[A_{n 0} P_{n}(\cos \theta)\right.\right. & \left.+\sum_{m=-n}^{n} B_{m}^{(n)} P_{n}^{|m|}(\cos \theta) e^{i m \phi}\right] \\
+ & \rho^{2} \sum_{\nu=1}^{\infty} \rho^{-2} b^{(\nu)}\left(\rho^{2}\right) \int_{-1}^{1}\left(1-\tau^{2}\right)^{n} \tau^{2 \nu} d \tau\left[A_{n 0} P_{n}(\cos \theta)\right. \\
& \left.\left.+\sum_{m=-n}^{n} B_{m}^{(n)} P_{n}^{|m|}(\cos \theta) e^{i m \phi}\right]\right) .
\end{aligned}
$$

(28) It should be stressed that in Theorem 4.1 of [6] we proved a similar result. We considered there however a sphere of a given radius, while in the present paper we consider only a sufficiently small neighborhood of the origin. 
If we write

$$
\begin{aligned}
& A_{n 0} \int_{-1}^{1}\left(1-\tau^{2}\right)^{n} d \tau=B_{0}^{(n, 0)}, \\
& B_{m}^{(n)} \int_{-1}^{1}\left(1-\tau^{2}\right)^{n} d \tau=B_{m}^{(n, \nu)},
\end{aligned}
$$

we obtain the formula (7.4) since $\rho^{-2} b^{(\nu)}\left(\rho^{2}\right)$ are entire functions of $\rho^{2}$.

TheOREM 7.1. The subsequence $\left\{B_{m}^{n, 0}\right\}$ of the coefficients of the series development (7.4) at the origin of a function $\psi(X) \in \mathfrak{Y}\left(a_{1}, \cdots, a_{p} ; N\right)$ satisfies the following conditions. There exist two real numbers $\phi_{1}$ and $\theta_{1},\left|\phi_{1}\right| \leqq \pi$, $\left|\theta_{1}\right| \leqq \pi / 2$, and an infinite sequence of numbers $\gamma_{n}$ which for $R \geqq R_{0}(p, N)$ satisfy the relation (6.4) and such that

$$
\begin{aligned}
(7.8 \mathrm{a}) & B_{0}^{(n, 0)} & =\gamma_{n+1} P_{n}\left(\cos \theta_{1}\right), \\
(7.8 \mathrm{~b}) & (n+\nu) !\left(B_{m}^{(n, 0)}+B_{-m}^{(n, 0)}\right) & =4 \gamma_{n+1}(n-\nu) ! P_{n}^{\prime}\left(\cos \theta_{1}\right) \cos \nu \phi_{1}, \\
(7.8 \mathrm{c}) & -i(n+\nu) !\left(B_{m}^{(n, 0)}-B_{-m}^{(n, 0)}\right) & =4 \gamma_{n+1}(n-\nu) ! P_{n}^{\prime}\left(\cos \theta_{1}\right) \sin \nu \phi_{1} .
\end{aligned}
$$

REMARK 7.2. In analogy to the two-dimensional case the conditions formulated in the Theorem 7.1 are independent of the coefficient $F$ of the equation (1.3).

Proof. According to Lemma 7.2 and the representation (7.7), a solution $\psi$ of the equation (1.3) is completely determined by the subsequence $\left\{B_{m}^{(n, 0)}\right\}$. By (7.3a), if $\psi \in \mathfrak{A}\left(a_{1}, \cdots, a_{p} ; N\right), \mathrm{p}_{3}^{-1}(\psi)$ must satisfy the conditions (6.3a), (6.3b), (6.3c). This yields our theorem.

In analogy to the second part of the Theorem 2.2 we obtain

TheOREM 7.2. If $\psi \in \mathscr{Y}\left(a_{1}, \cdots, a_{p} ; N\right)$, it can be represented in the form

$$
\psi(X)=\tilde{\widetilde{\psi}}(T, \rho)
$$

$$
=\frac{\rho^{1 / 2}}{2 \pi} \int_{\tau=-1}^{1} \Omega(\rho, \tau) \frac{d\left[\rho^{1 / 2} \int_{\zeta_{1}}^{\zeta_{2}} Q(\zeta) J\left(B^{\prime}+\frac{C}{2}, g_{2}, g_{3}\right) d \zeta\right]}{d \rho} d \tau
$$

where $J\left(s, g_{2}, g_{3}\right)$ is the inverse of the Weierstrass 8 -function and $T$ is given by (4.5a). Here

(7.10a) $\quad g_{2}=-4 B B^{\prime}+3 C^{2}$,

(7.10b) $\quad g_{3}=2 B C B^{\prime}-A B^{\prime 2}-C^{3}$

and

$$
\begin{aligned}
& A=-\rho^{2}\left(1-\tau^{2}\right)^{2}, \quad B=\left[2 R(\zeta)\left(1-\tau^{2}\right) \rho T+\rho^{2}\left(1-\tau^{2}\right)^{2}\right] / 4, \\
& C=-\left[R^{2}(\zeta)+2 R(\zeta)\left(1-\tau^{2}\right) \rho T\right] / 6, \quad B^{\prime}=R^{2}(\zeta) / 4 .
\end{aligned}
$$

Proof. It follows from (7.1a) and (7.2b) that $\psi$ can be represented in the 
form

$$
\begin{aligned}
\psi(X)= & \frac{2}{\pi} \int_{\tau=-1}^{1} \Omega(\rho, \tau) \rho^{1 / 2} \\
& \times\left\{d\left[\rho^{1 / 2} \int_{0}^{1} \tilde{G}\left(\rho t^{2}\left(1-\tau^{2}\right), \theta, \phi\right)\left(1-t^{2}\right)^{-1 / 2} d t\right] / d \rho\right\} d \tau .
\end{aligned}
$$

Since $\psi \in \mathfrak{A}\left(a_{1}, \cdots, a_{p} ; N\right)$ and $\mathbf{C}^{-3}(\psi)=\mathbf{C}^{-3}(G)$, according to the Definition $5.2,(4.4)$ and (4.5),

$$
\widetilde{G}(\rho, \theta, \phi)=\int_{\zeta_{1}}^{\zeta_{2}} \frac{Q(\zeta) d \zeta}{\left[\rho^{2}+R^{2}(\zeta)-2 R(\zeta) \rho T\right]^{1 / 2}}
$$

Thus in this case

$$
\int_{0}^{1}\left[\tilde{G}\left(\rho t^{2}\left(1-\tau^{2}\right), \theta, \phi\right)\right]\left(1-t^{2}\right)^{-1 / 2} d t
$$

$(7.14)=\int_{t=0}^{1} \int_{\zeta_{1}}^{\zeta_{2}} \frac{Q(\zeta) d \zeta}{\left\{\left[t^{4} \rho^{2}\left(1-\tau^{2}\right)^{2}-2 t^{2} R(\zeta) \rho\left(1-\tau^{2}\right) T+R^{2}(\zeta)\right]\left(1-t^{2}\right)\right\}^{1 / 2}} d t$

$(7.14 \mathrm{a})=\frac{1}{2} \int_{\zeta_{1}}^{\zeta_{2}} Q(\zeta)\left[\int_{x=0}^{1} \frac{d x}{\left(A x^{4}+4 B x^{3}+6 C x^{2}+4 B^{\prime} x\right)^{1 / 2}}\right] d \zeta$

where $A, B, C, B^{\prime}$ are functions of $\rho, T, \tau, \zeta$ introduced in (7.11).

By the transformation $s(x)=B^{\prime} x^{-1}+C / 2$, see I, p. 13 and III, p. 14 of [20], the interior integral in (7.14a) goes into

$$
-\int_{\infty}^{B^{\prime}+C / 2} \frac{d s}{\left(4 s^{3}-g_{2} s-g_{3}\right)^{1 / 2}}=J\left(B^{\prime}+\frac{C}{2}, g_{2}, g_{3}\right)
$$

see p. 229 of [20].

Substituting this expression for the interior integral in (7.14a) and replacing the interior integral in (7.12) by the new expression we obtain (7.9).

8. Concluding remarks. As we stressed in the introduction, the interest of our considerations is not merely in the isolated results formulated in the paper, but rather in establishing methods of exploiting the laws which connect different classes of functions obtained with the help of mappings by integral operators of the first kind. In this connection it is of interest to use also other integral operators. In particular, as has been shown in [5], the representation of harmonic functions with the help of the fundamental solution can be employed to define a mapping by an integral operator. Using the fundamental solution of

$$
\Delta \psi+F\left(x_{1}, x_{2}, x_{3}\right) \psi=0
$$


see [14b], a mapping analogous to (4.4) of polynomials $Q$ into solutions of equation (8.1) can be introduced. This mapping permits us to extend a large part of the results of [5] to the case of differential equations $\left({ }^{29}\right)(8.1)$.

A further type of integral operator which is valuable for the study of certain types of differential equations (8.1) is that for which the generating function $E$ can be represented in the form

$$
E=P(X, t) \log Q(X, t), \quad \boldsymbol{X}=\left(x_{1}, x_{2}, x_{3}\right),
$$

where $P$ and $Q$ are polynomials in $t$. (Procedures used in $[2, \S 3]$ can be repeated in the three-dimensional case to a large extent.)

In some instances singularities of differential equations in three variables can be obtained by reducing them by a convenient substitution to equations in two variables, e.g., the substitution $\Psi(x, y, z)=\cos \nu z \psi(x, y)$ reduces $\Psi_{x x}+\Psi_{y y}+\Psi_{z z}=P(x, y) \Psi$ to $\psi_{x x}+\psi_{y y}=\left(\nu^{2}+P(x, y)\right) \psi$.

The relations between the coefficients of the function element of $\psi$ and the property of the solution $\psi$ obtained by the use of integral operators different from those of the first kind in most instances depend upon the coefficient $F$ so that we are led by these operators to new types of theorems.

It should be mentioned also that similar to our results, relations between the subsequence $\left\{a_{m \nu}\right\}, m=0,1,2, \cdots ; \nu>0$, of the coefficients of the function element $\psi=\sum a_{m n} z^{m} z^{* n}$ of the solution $\psi$ and its properties can be established, see [15].

The functions considered in the present paper represent the simplest class of solutions of (1.1) for which representations in terms of classical functions can be obtained. (See Theorem 2.2.) It is known (see $[14, \$ \$ 14-16]$, $[14 \mathrm{a}, \S \S 14,16])$ that if a function $g$ is a solution of an ordinary linear differential equation

$$
\sum_{\nu=0}^{n} P_{n-\nu}(z) g^{[\nu]}=0, \quad g^{[\nu]}=d^{\nu} g / d z^{\nu},
$$

where $P_{\mu}(z)$ are algebraic functions of $z$ satisfying certain conditions, the integrals $\int_{0}^{z}(z-\zeta)^{n-1} g(\zeta) d \zeta$ and $\int_{-1}^{1} g\left(z\left(1-t^{2}\right)\right) d t / t^{2}$ can be represented by automorphic $\theta$-functions and related functions. Using these results, one obtains representations analogous to those indicated in Theorem 2.2 for solutions of (1.1) whose C-associates (see p. 2) satisfy equations of type (8.3).

Similar theorems can be obtained also in the three-dimensional case for certain solutions of (1.3). See also $[1, \S 5]$, in particular p. 551.

Thus one sees that the method of integral operators opens various and fruitful possibilities in such a comparatively little explored field as the theory of multivalued solutions of linear partial differential equations.

(29) It should be mentioned that the method of the integral operator of the first kind can be extended to the general equation (8.1) as will be shown in another place. Since some additional complications arise in this connection we do not discuss this case in the present paper. 


\section{BIBLIOGRAPHY}

1. Stefan Bergman, Zur Theorie der algebraischen Potentialfunktionen des drei-dimensionalen Raumes, Math. Ann. vol. 99 (1928) pp. 629-659, and vol. 101 (1929) pp. 534-558.

2. — Zur Theorie der Funktionen, die eine lineare partielle Differentialgleichung befriedigen, Rec. Math. (Mat. Sbornik) N.S. vol. 2 (1937) pp. 1169-1198.

3. - Linear operators in the theory of partial differential equations, Trans. Amer. Math. Soc. vol. 53 (1943) pp. 130-155.

4. - Certain classes of analytic functions of two real variables and their properties, Trans. Amer. Math. Soc. vol. 57 (1945) pp. 299-331.

5. - A class of harmonic functions in three variables and their properties, Trans. Amer. Math. Soc. vol. 59 (1946) pp. 216-247.

6. - Classes of solutions of linear partial differential equations in three variables, Duke Math. J. vol. 13 (1946) pp. 419-458.

7. - Operator methods in the theory of compressible fluids, Proceedings of Symposia in Applied Mathematics, American Mathematical Society, vol. 1, 1949, pp. 19-40.

8. - On solutions with algebraic character of linear partial differential equations, Trans. Amer. Math. Soc. vol. 68 (1950) pp. 461-507.

9. - On solutions of linear partial differential equations of mixed type, Amer. J. Math. vol. 74 (1952) pp. 444-474.

10. L. Bieberbach, Lehrbuch der Funktionentheorie, vol. 2, Leipzig, Berlin, 1931.

11. M. M. E. Eichler, On the differential equation $u_{x x}+u_{y y}+N(x) u=0$, Trans. Amer. Math. Soc. vol. 65 (1949) pp. 259-278.

12. - Analytic functions in three-dimensional Riemannian spaces, Duke Math. J. vol. 16 (1949) pp. 339-349.

13. P. Frank and R. v. Mises, Die Differential- und Integralgleichungen, 2d ed., vol. 1, 1943.

14. R. Fricke, Automorphe Funktionen mit Einschluss der elliptischen Modulfunktionen, Encyklopädie der Mathematischen Wissenschaften II B. 4, Leipzig, 1921.

14a. E. Hilb, Lineare Differentialgleichungen im komplexen Gebiet, Encyklopädie der Mathematischen Wissenschaften II B. 5, Leipzig, 1921.

14b. E. Holmgren, Über die Existenz der Grundlösung bei einer linearen partiellen Differentialgleichung der zweiten Ordnung vom elliptischen Typus, Arkiv för Mathematik, Astronomi och Fysik vol. 1 (1903-1904) pp. 209-224.

15. B. M. Ingersoll, The regularity domains of solutions of linear partial differential equations in terms of the series development of the solution, Duke Math. J. vol. 15 (1948) pp. 10451056.

16. M. Marden, A recurrence formula for the solutions of certain linear partial differential equations, Bull. Amer. Math. Soc. vol. 50 (1944) pp. 208-217.

17. J. Mitchell, Some properties of solutions of partial differential equations given by their series development, Duke Math. J. vol. 13 (1946) pp. 87-104.

18. K. L. Nielsen, Some properties of functions satisfying partial differential equations of elliptic type, Duke Math. J. vol. 11 (1944) pp. 121-137.

19. K. Weierstrass, Vorlesungen über die Theorie der Abelschen Transcendenten, Mathematische Werke, vol. 4, Berlin, 1902.

20. - Vorlesungen ïber die Theorie der Elliptischen Funktionen, Mathematische Werke, vol. 5, Berlin, 1915.

21. A. Zygmund, Trigonometrical series, Warsaw, 1935.

HARVARD UNIVERSITY, Cambridge, Mass. 